Article

\title{
Development of a Butterfly Wind Turbine with Mechanical Over-Speed Control System
}

\author{
Yutaka Hara ${ }^{1, *}$, Kotaro Tagawa ${ }^{2}$, Shigenori Saito ${ }^{3}$, Keisuke Shioya ${ }^{3}$, Takeshi Ono ${ }^{3}$, \\ Kenji Makino ${ }^{4}$, Kazutoshi Toba ${ }^{4}$, Takakazu Hirobayashi ${ }^{5}$, Yousuke Tanaka ${ }^{5}$, \\ Kazuo Takashima ${ }^{6}$, Susumu Sasaki ${ }^{6}$, Kengo Nojima ${ }^{7}$ and Shigeo Yoshida ${ }^{8}$ (d)
}

1 Faculty of Engineering, Tottori University, 4-101 Koyama-Minami, Tottori 680-8552, Japan

2 Faculty of Agriculture, Tottori University, 4-101 Koyama-Minami, Tottori 680-8552, Japan;

tagawa@tottori-u.ac.jp

3 Nikkeikin Aluminium Core Technology Co., Ltd., NYK Tennoz Building, No.2-20, 2-chome,

Higashi-shinagawa, Shinagawa, Tokyo 140-0002, Japan; shigenori-saito@act.nikkeikin.co.jp (S.S.);

keisuke-shioya@act.nikkeikin.co.jp (K.S.); take_c.ono@nifty.ne.jp (T.O.)

4 Enatex Co., Ltd., 2-73 Seidani-cho, Kurayoshi, Tottori 682-0017, Japan; makino@enatex.co.jp (K.M.); toba@enatex.co.jp (K.T.)

5 SKY Electronics Co., Ltd., 1380-56, Higashionaro, Shimanto-cho Takaoka-gun, Kochi 786-0027, Japan; hiro@sky-denshi.co.jp (T.H.); tanaka@sky-denshi.co.jp (Y.T.)

6 Japan Micro System Co., Ltd., 28-1 Takashima, Yonago, Tottori 689-3536, Japan; kazuo@jpms.co.jp (K.T.); susumu@jpms.co.jp (S.S.)

7 Tottori Institute of Industrial Technology, 1247 Kusaka, Yonago, Tottori 689-3522, Japan; nojima-k@tiit.or.jp

8 Research Institute for Applied Mechanics, Kyushu University, 6-1 Kasugakoen, Kasuga, Fukuoka 816-8580, Japan; yoshidas@riam.kyushu-u.ac.jp

* Correspondence: hara@tottori-u.ac.jp; Tel.: +81-857-31-6758

Received: 21 May 2018; Accepted: 8 June 2018; Published: 12 June 2018

\begin{abstract}
Based on a concept of "cost reduction by large rotor and small generator", a kind of small vertical axis wind turbine (VAWT) called a butterfly wind turbine (BWT) has been developed with rotor diameter of $7 \mathrm{~m}$ and five looped blades. One of the features is a mechanical system which can twist each blade of a VAWT by using the centrifugal force acting on the blades in order to prevent over-speeding of the rotor. An electric power generation system that combines the BWT utilizing the over-speed control system (OCS) with a coreless generator connected to a three-time increasing gear unit is constructed in this study. The performance of the BWT power-generation system is predicted by the blade element momentum (BEM) theory. The final goal of this research is to show the feasibility of the low-cost VAWT power generation system with the proposed OCS. From the results of a series of forcible rotation experiments of the BWT rotor that is driven by an induction motor in very low wind speed condition, it is shown that the twist movement of the blades is improved by the design of the OCS that considers the observed radial force deprived of the centrifugal force and the pre-compression of springs. In the generation experiments with the final design of the OCS, the expected twists of the blades are observed for the first time in strong natural wind speed over $18 \mathrm{~m} / \mathrm{s}$.
\end{abstract}

Keywords: wind-power generation; vertical axis wind turbine; over-speed control system; renewable energy; cost reduction; small-size wind turbine; dryland

\section{Introduction}

The cost of wind-power generation has been gradually reduced by enlarging the size of wind-turbine rotors and now wind has become one of the main power sources in the world. The global cumulative installed capacity of wind power has been growing and exceeded $539 \mathrm{GW}$ at the end 
of 2017 [1]. However, the introduction of large-size wind turbines often faces problems such as concerns about spoiling the scenery and hurting the natural environment. Therefore, time-consuming environmental research is needed before introduction of large-size wind turbines [2]. On the other hand, small-size wind turbines can be installed easily in various places due to their less important impact on the environment and easy transportation. Therefore, small-size wind power generation is expected to become a clean energy source at various places where it is difficult to introduce large-size wind turbines due to specific reasons or where a site is not suitable for photovoltaic power generation. In particular, the demand for small wind power systems might be large at high latitudes that are abundant in wind energy compared to solar energy and in drylands in which electricity is not used sufficiently in people's daily lives. However, since the wind energy captured by the small rotor is not large, generally speaking, the cost of energy of a small wind-power system is expensive and the market has not grown in comparison with large-size wind turbines.

Changing the viewpoint, although the mainstream of wind power is large-size propeller-type horizontal axis wind turbines (HAWTs), small-size vertical axis wind turbines (VAWTs) have the possibility to be suitable for installation in places near a living environment or to lead to cost reduction due to the simple structure and non-dependence on wind direction. Therefore, many studies on small VAWTs have been conducted recently [3-5].

A series of studies on the cost reduction of small-size wind turbines was started in Tottori University in 2009. In that study, a VAWT which featured an armless rotor, i.e., a rotor without spokes, with looped blades was invented. The VAWT was named the "Butterfly Wind Turbine (BWT)" as the rotor is shaped like a butterfly [6]. The BWT would be expected to have small aerodynamic resistance and small blade-tip loss due to the armless structure. The shape of the BWT was originally considered to have a generator installed near the center of the rotor and its rotational axis could be short in order to avoid large vibration. Although the blades of experimental wind turbines were initially made of plastics or composite materials, adoption of aluminum blades made by extrusion molding gave the possibility of mass production and cost reduction of the BWT [7].

In the project assigned by Tottori prefecture to Tottori University, Japan Micro System, SKY Electronics, Enatex, and Nikkeikin Aluminium Core Technology (28 January 2016 to 27 January 2018), a BWT of $7 \mathrm{~m}$ diameter has been developed based on a concept of "cost reduction by large rotor and small generator" [8]. This concept means increasing the performance of the wind-turbine rotor against the capacity of the generator in order to obtain a high capacity factor even at a low wind site. For a small-size wind turbine, the costs of electric devices including a generator as the main part are relatively expensive compared to other parts. A relatively large turbine rotor consisting of aluminum blades made by extrusion can gather much wind energy cost-effectively. One of the features of the BWT developed in the project is a mechanical system which can twist each blade of the turbine by using the centrifugal force acting on the blades so as to prevent over-speed of the rotor. An over-speed control system (OCS) is important for wind turbines to increase safety and reduce the cost of energy. Various methods to prevent the over-speed state of HAWTs such as furling or pitch-control of the blades have been applied to commercial wind turbines [9]. However, most OCS for VAWTs are in the experimental stage [10]; there are very few OCS at practical level due to the problems of durability or cost.

The purpose of this study is to prove the feasibility of the OCS for VAWT proposed here. In this paper, the final design of the OCS, which can realize movements similar to theoretical prediction as the result of improvements through the two-year project, is explained. Forcible rotation experiments, which had been carried out in a previous three experiment-series with a different version of the OCS, are carried out by using a geared-induction motor in a low wind-speed state for the BWT equipped with the final-version of the OCS. The details of the power-generation control system developed in the project are shown. The electric power-generation experiments were continued after the project assigned by Tottori prefecture had expired. The results of the experiments of electric generation are investigated by comparison with theoretical predictions, and the first observation of the twisting of blades with the OCS in operation in the strong natural wind is reported. 


\section{Experimental Wind-Turbine System}

This section includes the following descriptions: the specifications and the characteristics of the wind-turbine rotor (Section 2.1), the mechanism, final design, and movement prediction of the OCS (Section 2.2), the geared generator and the performance prediction of the BWT (Section 2.3), the power-generation control system (Section 2.4), and the site of the experiments (Section 2.5). It should be noted that the prediction described in Section 2.3 about the BWT's performance is a little different from that reported previously for the first experiment series of forcible rotations (implementation: 22 December 2016) [8] because of modifications to the parameters used in the prediction code in addition to the alteration of the design of the OCS.

\subsection{Wind-Turbine Rotor}

Figure 1 shows the prototype of the BWT equipped with an OCS for five looped blades. Two photographs in Figure 1 were taken in the second experiment series of forcible rotations (implementation: 25 July 2017); the left-hand photograph (a) shows the no-twist state, in which the main straight parts of blades are in the vertical condition, and the right-hand photograph (b) shows the twist state, in which the twist angle $\eta$ is 30 degrees. Those photographs are used in Figure 1 in order to illustrate the main feature of the wind turbine developed in this study, even though the second experiment series of forcible rotations was not successful because a great decrease in rotational speed and large time lag were observed during the process of the twisted blades returning to the original no-twist state. In the forcible rotation experiments, the turbine rotor was equipped with an induction motor instead of a generator. The diameter of the rotor $D$ was $7.0 \mathrm{~m}$ and the height $H$ was $2.7 \mathrm{~m}$. The specification of the BWT rotor is given in Table 1.

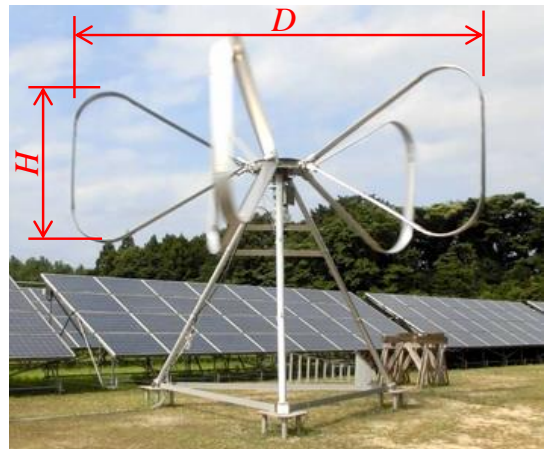

(a)

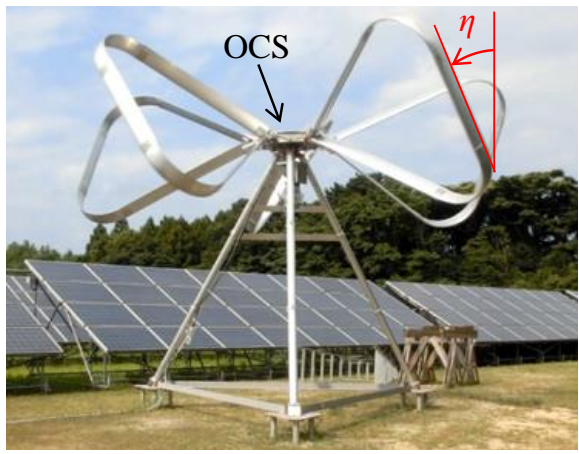

(b)

Figure 1. Prototype of the butterfly wind turbine (BWT) equipped with an over-speed control system (OCS) for 5 looped blades. The photographs shown above were taken during the second experiment-series of forcible rotation by an induction motor under conditions of low wind speed (about $1 \mathrm{~m} / \mathrm{s})$ : (a) no-twist state; (b) twist state ( $\eta=30 \mathrm{deg}$.).

Table 1. Specifications of the BWT rotor.

\begin{tabular}{ccc}
\hline Characteristic & Symbol & Value or Matter \\
\hline rotor radius & $R$ & $3.5 \mathrm{~m}$ \\
rotor height & $H$ & $2.7 \mathrm{~m}$ \\
chord length & $c$ & $0.242 \mathrm{~m}$ \\
number of blade & $B$ & 5 \\
blade section & - & NACA 0018 \\
swept area & $A_{0}$ & $18.6 \mathrm{~m}^{2}$ \\
solidity & $\sigma(=B c / 2 \pi R)$ & 0.055 \\
blade material & - & Aluminum \\
weight of a blade & - & $36.3 \mathrm{~kg}$ \\
\hline
\end{tabular}


The OCS was located at the rotor center, or hub position, of the wind turbine and the height from the ground level was $4.3 \mathrm{~m}$. As mentioned before, the concept of development was "cost reduction by large rotor and small generator" and the goal was cost reduction by enlarging the turbine rotor using cost-effective extruded aluminum blades and, on the other hand, by adopting a small-capacity generator and other electric devices with small capacity, which tend to become expensive depending on their capacity. As mentioned, cost reduction could also be contributed by the mechanical OCS, since it can increase the capacity factor by continuing the electric generation during any strong wind conditions without stopping the wind turbine.

The prototype of the BWT was originally planned to be installed on the top of a high-rise building to obtain large wind energy. All the parts of the BWT, including the blades in a disassembled state, can be carried to the highest floor by using the elevators of the buildings. It is one of the features of the prototype that a rope made of high tenacity polyarylate fiber $(\varphi 10 \mathrm{~mm}$, breaking load: $53.9 \mathrm{kN})$ is inserted into each blade and all the five ropes are connected each other at the hub of rotor in order to prevent the scattering of fragments of broken blades in case of damage to the turbine rotor.

Because the BWT structure enables the generator to be installed close to the center of the rotor, the rotational axis of the wind turbine becomes short. The length between the rotor center and the first bearing is $0.158 \mathrm{~m}$ in the case of the prototype.

As shown in Figure 1, the stand consists of three legs which support the stand-top part located just below the hub of the rotor. The tripod structure gives the stand robustness so as not to vibrate against the exciting force of the rotor. Each base plate of the legs was anchored to the ground by three ground screws (length: $1.6 \mathrm{~m}$ ) instead of an expensive concrete foundation.

\subsection{Over-Speed Control System (OCS)}

Figure 2 is a photograph of the improved OCS. Five units are connected to a synchronizing disk located at the center of rotor hub by five links to obtain the same movements for all the blades. Universal joints configure the link portion and are important parts for realizing the blades' twist. The universal joints used in the previous experiments were commercial products and were made of high-strength zinc alloy. However, due to the stress levels and the lack of strength, they were exchanged by trial products (Matsumura Seiki, Inc., Tottori, Japan) made of stainless steel.

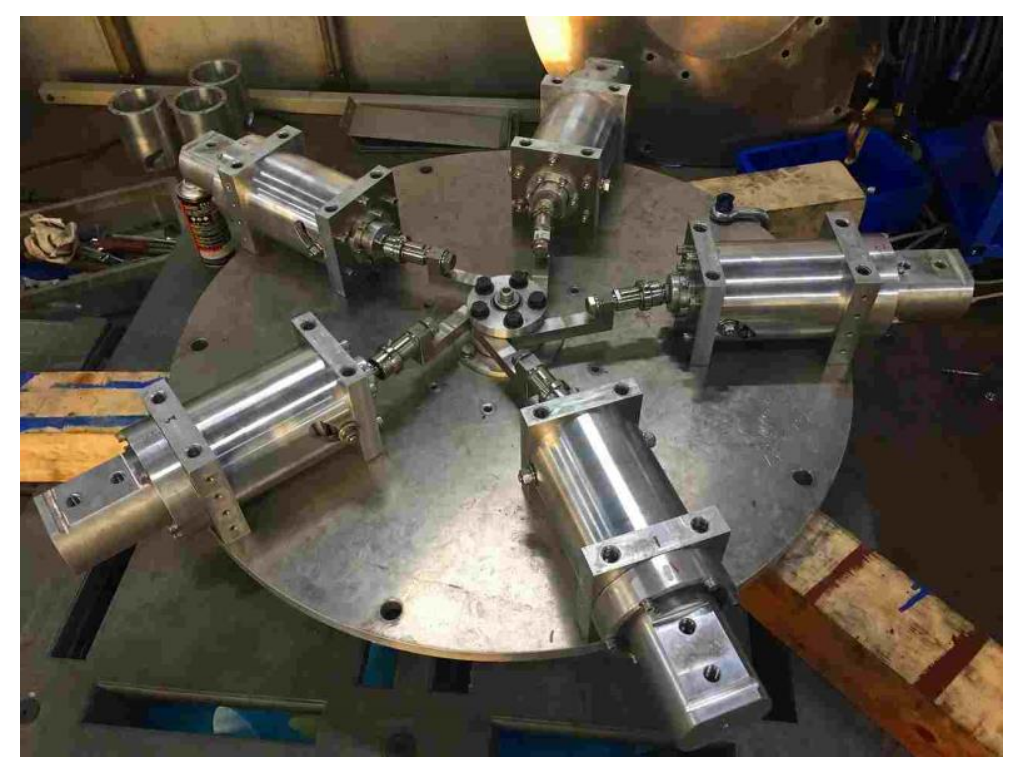

Figure 2. OCS for the prototype BWT with 5 blades. 
The schematic diagram of an OCS unit is illustrated in Figure 3. Centrifugal force $F_{c}$ acting on a blade moves a blade axis (max. diameter: $90 \mathrm{~mm}$, min. diameter: $50 \mathrm{~mm}$ ) inside a cylindrical frame in the radial direction ( $x$ direction). By this movement, a pin $(\varphi 20 \mathrm{~mm})$ jutting out on both sides from the blade axis moves along a pair of guide grooves, each of which consists of a straight portion and a helical portion.

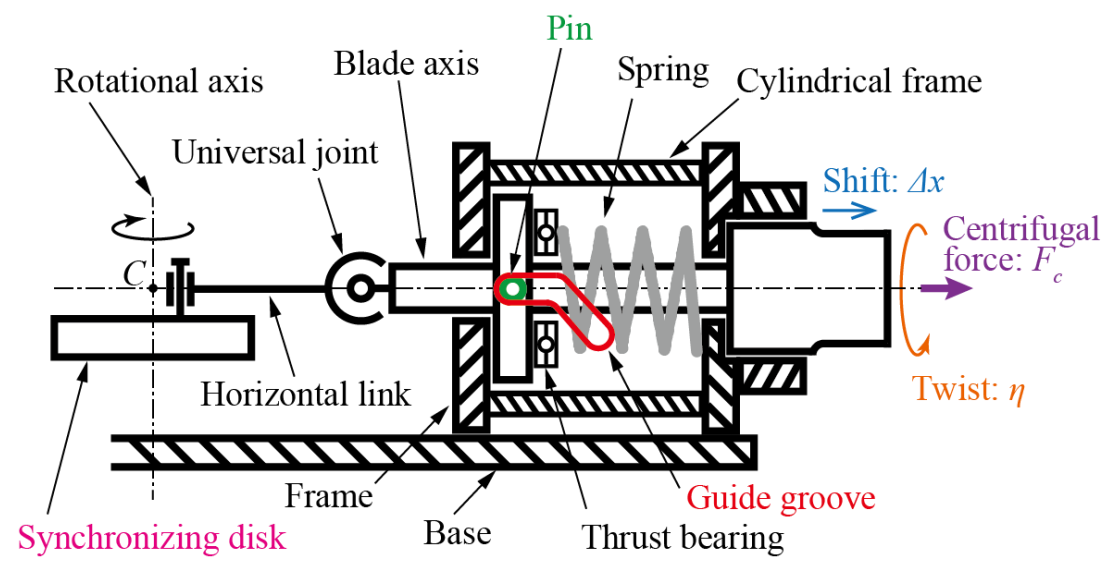

Figure 3. Schematic diagram of the OCS unit and synchronizing system.

Figure 4 is the schematic diagram to illustrate the relation between the rotational angle $\Delta \xi$ of the synchronizing disk and the displacement $\Delta x$ of the blade axis, which is the same as the shrinkage of a spring from the initial length. In the OCS, the length of each spring is $115 \mathrm{~mm}$ under the initial condition, or stationary condition, of the turbine rotor, and the centrifugal force $F_{c}$ is equal to zero in that state. In the initial condition, the distance between the pivot of a universal joint and the center $C$ of the synchronizing disk is $x_{0}=184.5 \mathrm{~mm}$; the angle between the line segment $(r=40 \mathrm{~mm})$, which connects the center $C$ of the synchronizing disk and one end of the horizontal link (length: $l=179 \mathrm{~mm}$ ), and the direction of the blade axis ( $x$ direction) is $\xi_{0}=75.89^{\circ}$.

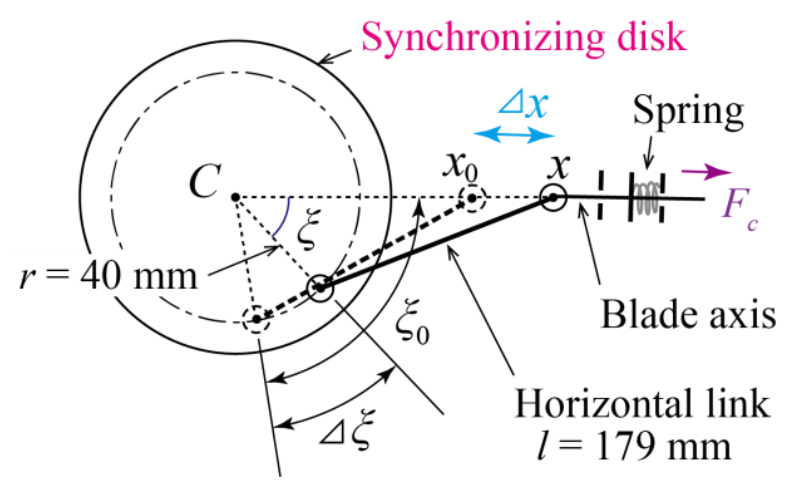

Figure 4. Schematic diagram illustrating relation between the displacement $\Delta x$ and the rotational angle $\Delta \xi$ of the synchronizing disk.

Until the third experiment series of forcible rotations (implementation: 31 August 2017), the natural length of the springs was $115 \mathrm{~mm}$. The natural length of the springs used in the fourth experiment series of forcible rotations (implementation: 15 December 2017) was $125 \mathrm{~mm}$ in order to generate an initial restoring force. In advance, the springs were compressed by $10 \mathrm{~mm}$ so as to be the initial length of $115 \mathrm{~mm}$ in the stationary condition of the rotor. This improvement eliminates the lack of stroke length of blade axes and contributes to diminishing the inclination of the helical portion of guide grooves. The constant of springs used in the final experiment-series was $k=548 \mathrm{~N} / \mathrm{mm}$. 
The rotational angle $\Delta \xi$ [rad] of the synchronizing disk and the displacement $\Delta x$ [mm] of the blade axis are given by the following Equations, from (1) to (3):

$$
\begin{gathered}
\xi=\xi_{0}-\Delta \xi, \\
x=r \cos \xi+\sqrt{r^{2} \cos ^{2} \xi+l^{2}-r^{2}}, \\
\Delta x=x-x_{0} .
\end{gathered}
$$

The relation between the displacement (shrinkage) $\Delta x[\mathrm{~mm}]$ of a spring from the initial length and the restoring force $F_{s}[\mathrm{~N}]$ is expressed by Equation (4):

$$
F_{s}=k \Delta x+F_{p}=548 \Delta x+5480,
$$

where $F_{p}$ is the initial restoring force by the pre-compression of the spring. Equation (4) means that a pin does not move along the guide grooves until the centrifugal force acting on a blade exceeds $5480 \mathrm{~N}$.

Considering the displacement $\Delta x$, the centrifugal force $F_{c}$ acting on a blade when the turbine rotor revolves at an angular velocity $\omega$ is given by the following Equation (5):

$$
F_{c}=m_{b}\left(x_{b}+\Delta x\right) \omega^{2},
$$

where $m_{b}$ is the total mass of a blade including the blade axis, connection parts, bolts, nuts, and so on. $x_{b}$ is the $x$-coordinate of the center of mass of the blade based on the rotational center $C$ under stationary condition.

Since the aerodynamic force acting on the blades in the radial direction was predicted to be much smaller than the centrifugal force when wind speed was not very large, the OCS was designed by considering only the centrifugal force until the third experiment series of forcible rotations. However, as shown in Figure 5, which indicates the observed results of the third forcible rotation experiments, the behavior of the blade-axis displacement $\Delta x$ was different from the prediction obtained by considering only the centrifugal force at the conditions of the rotor rotational speed of $80 \mathrm{rpm}$ or more, when the blades were greatly twisted. This means the existence of large force in radial direction except for the centrifugal force, but the cause of this phenomenon has not been made clear yet. As shown in Figure $5 \mathrm{a}, \mathrm{b}$, it is clear that the force in question is produced in association with the blade twist. Large twist moment might be generated by some causes, such as the deformation of the blades or the unexpectedly large distance between the aerodynamic center and the supporting point of a blade.

Figure 6 shows the relations, which were obtained from the selected data in the third experiment series of forcible rotations, between the radial force $F_{t w}$ deprived of the centrifugal force and the twist angle $\eta$. A line in black in Figure 6 is the approximation curve, which is expressed by Equation (6):

$$
F_{t w}=-25.466 \eta^{2}+888.97 \eta-1039.1
$$

which is valid at $\eta>1.211^{\circ}$ and when $\eta$ is less than $1.211^{\circ}$ the force in question is assumed to be zero $\left(F_{t w}=0\right)$.

Under the condition of constant rotor rotation, the equilibrium of forces acting on a blade axis in the radial direction is assumed to be $F_{c}+F_{t w}=F_{s}$. From this assumption, the displacement $\Delta x$ is given as a result of the following Equation:

$$
\Delta x=\frac{m_{b} \omega^{2} x_{b}-F_{p}+F_{t w}}{k-m_{b} \omega^{2}} .
$$




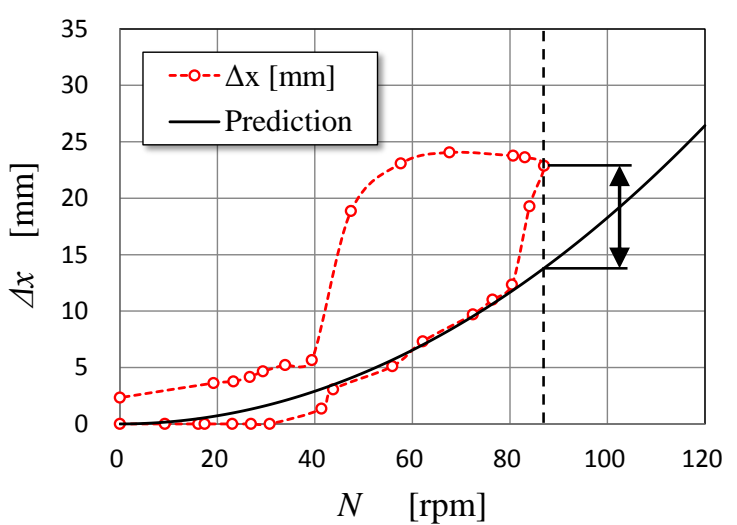

(a)

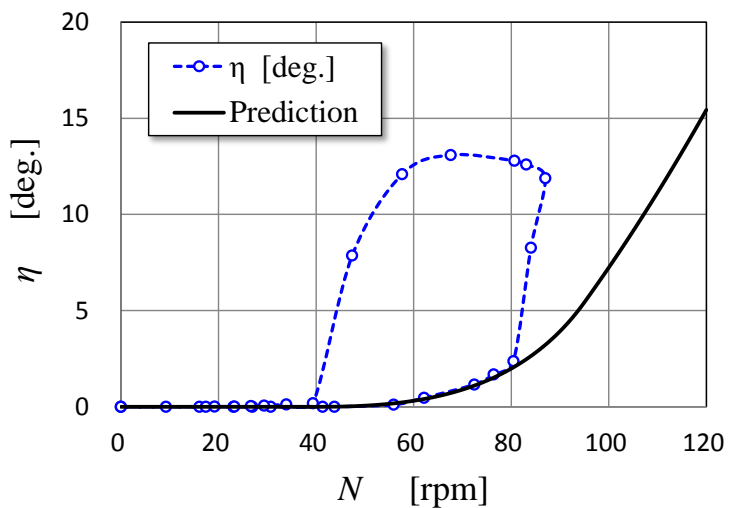

(b)

Figure 5. Phenomena observed in the third experiment series of forcible rotation: (a) relation between displacement $\Delta x$ and rotational speed $N$; (b) relation between twist angle $\eta$ and rotational speed $N$. Each line in black indicates the prediction which is obtained based on only the consideration of centrifugal force acting on a blade. Therefore, the differences (illustrated by a two-directional arrow) between the experimental data and the predictions observed over $80 \mathrm{rpm}$ imply the effects of another force, the cause of which has not been made clear yet. However, that force probably should be associated with the twist angle.

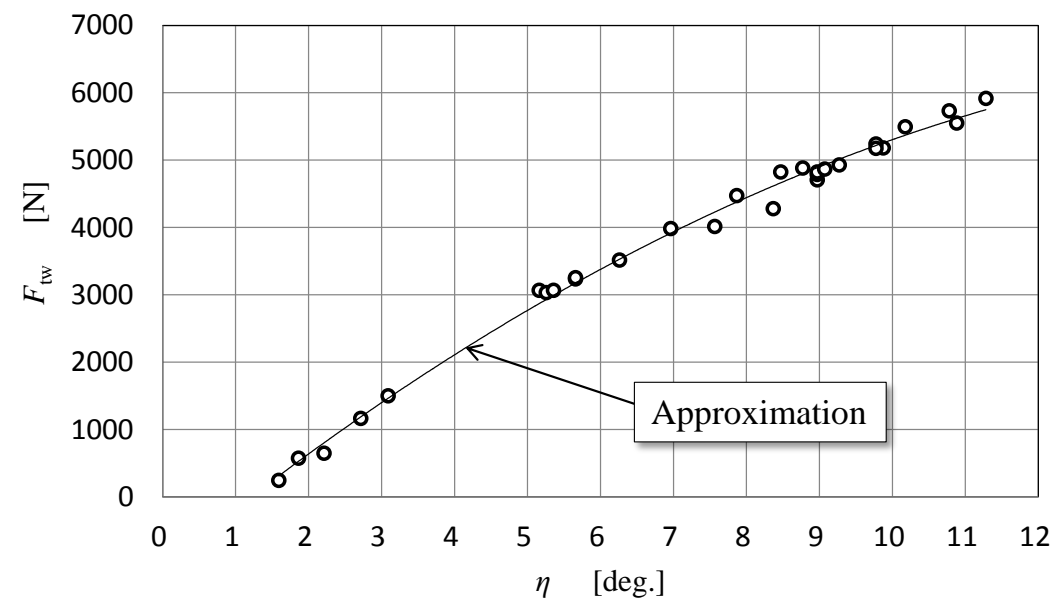

Figure 6. Relations, obtained from the selected data measured in the third experiment series of forcible rotations, between the twist angle $\eta$ and the radial force $F_{t w}$ deprived of the centrifugal force. The line in black shows the approximate curve.

By using Equation (7), when the shape of a guide groove is given, the displacement $\Delta x$ and also the twist angle $\eta$ can be decided for an arbitrary rotor rotational speed $N=30 \omega / \pi[\mathrm{rpm}]$. Although the force $F_{t w}$ is a function of $\eta$ (see Equation (6)), in the calculation process of $\Delta x$ and $\eta$ from the stationary state $\left(N=0 ; F_{t w}=0\right)$ with a small interval of rotational speed $\Delta N$, the value of $F_{t w}$ obtained from the preceding calculation can be substituted for the value of $F_{t w}$ in the next step of the calculation.

Figure 7 is an illustration of a cylindrical frame with the guide grooves of the final design, with which the pre-compression of springs is applied. Two guide grooves are formed at the positions of the rotational symmetry with respect to the center line of the cylindrical frame. A guide groove has a straight portion $(4 \mathrm{~mm})$ and a helical portion. The angle between the center line of the helical portion and the center line of the cylindrical frame is $\zeta=46.73^{\circ}$, when it is considered with an expansion plan. The inclination angle $\zeta$ of the final design becomes small compared to the previous designs of the guide grooves. In addition, the maximum blade twist angle that can be realized by this final design is $\eta=18^{\circ}$. 


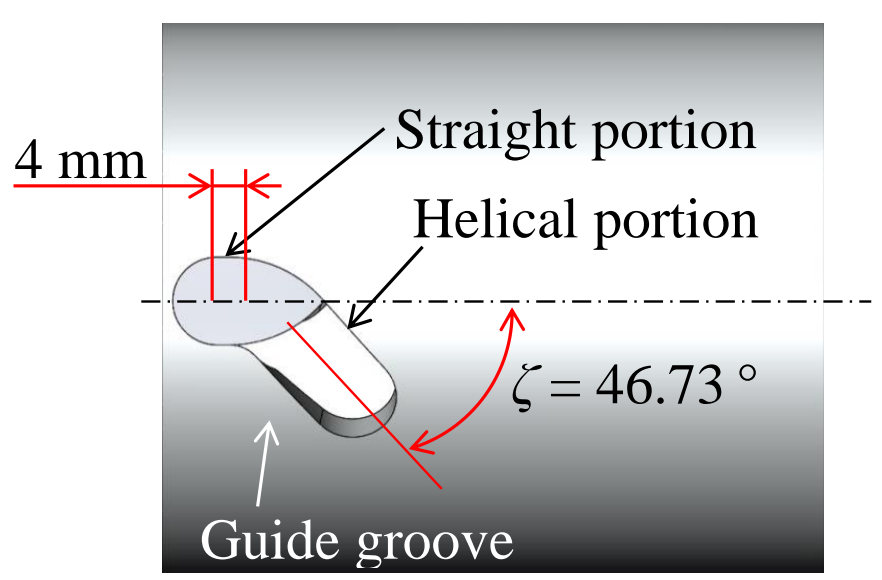

Figure 7. The final design of the guide grooves which are formed in a cylindrical frame. Two guide grooves are formed at the positions of rotational symmetry with respect to the center line of the cylindrical frame.

Figure 8 shows the prediction based on the final shape of the guide grooves about the dependence of the displacement and the twist angle on the rotational speed. The prediction shows that the blades begin to be twisted at the rotational speed of around $90 \mathrm{rpm}$. Since the twist angle becomes large abruptly when the rotational speed exceeds $110 \mathrm{rpm}$ according to Figure 8, the rated rotational speed of the BWT after this study is assumed to be $110 \mathrm{rpm}$.

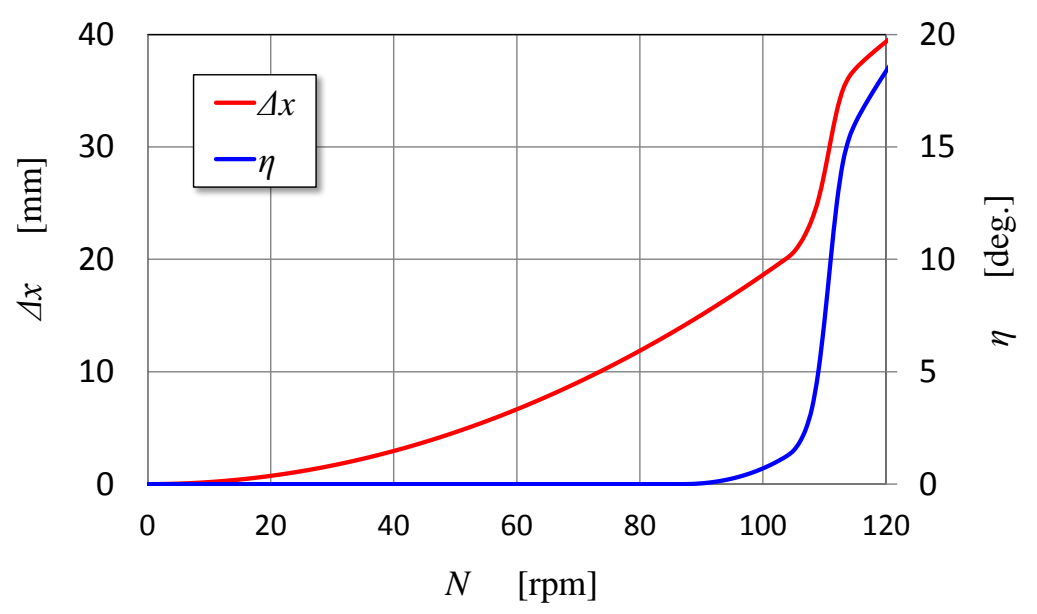

Figure 8. Predictions of displacement $\Delta x$ and twist angle $\eta$ when the improved OCS is operated properly.

\subsection{Generator and Prediction of the Performance of the Butterfly Wind Turbine (BWT)}

The generator installed in the BWT is shown in Figure 9. That is a permanent magnet-type multi-pole generator (SKY Electronics Co., Ltd., Shimanto-cho, Japan, MG450W, 5 kW, 350 rpm, 48 poles) and is coreless and inner rotor type. A speed-increasing planetary gear (Matex Co., Ltd., Yao, Japan, LGU-200, 3:1) is combined with the generator. As shown in Figure 9, the planetary gear is connected to the bottom of the stand-top part, in which there are three bearings to hold the rotational axis (max. diameter: $90 \mathrm{~mm}$ ) at the center. The stand-top part has three attachment plates for connection with the tripod legs. 


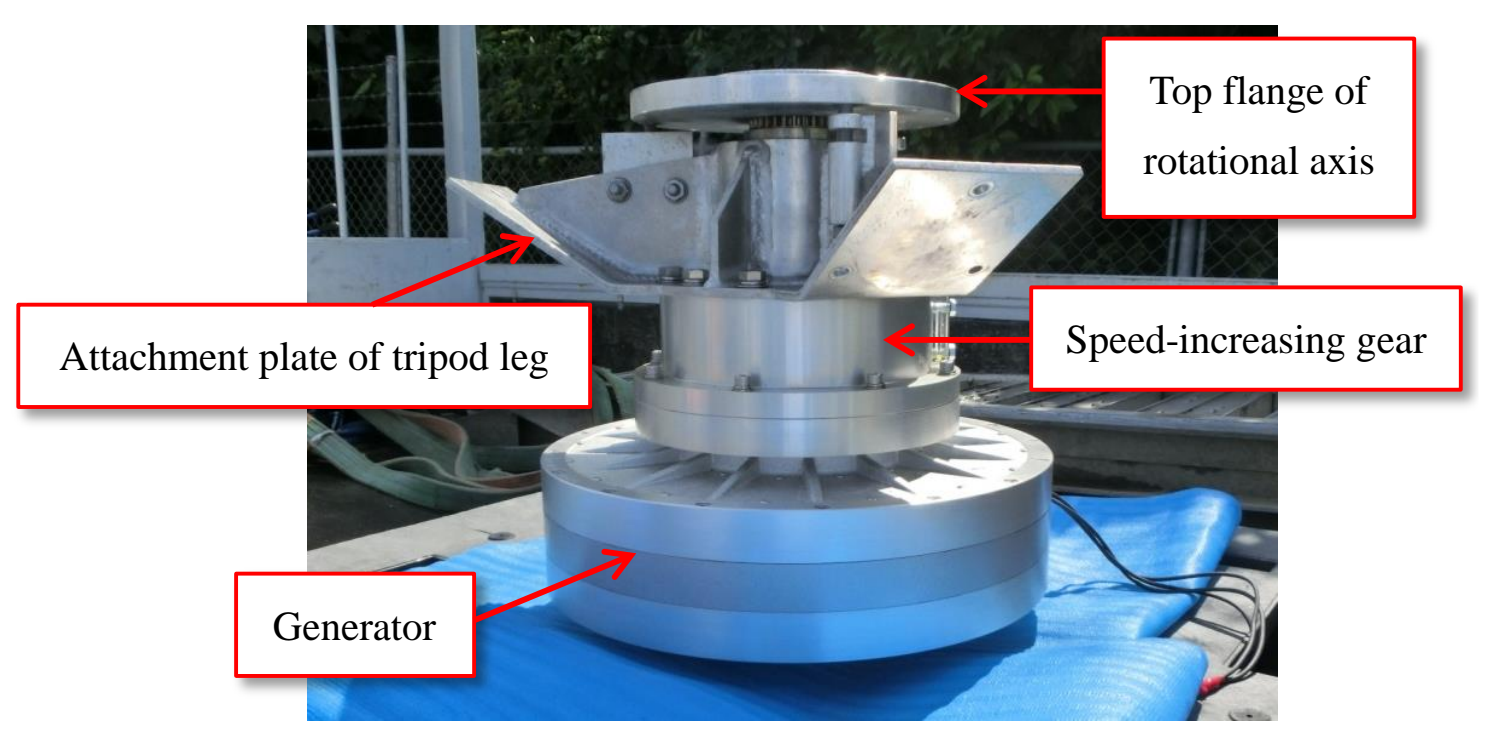

Figure 9. Coreless generator MG450W equipped with a speed-increasing gear LGU-200.

The performance of the wind turbine was predicted by the blade element momentum (BEM) theory [11] in this study. The rotor of the BWT has a double-blade structure, which means that fluid flowing into the rotor swept area $A$ has the possibility to intersect the blades four times. Therefore, in the prediction based on the BEM theory, the quadruple-multiple streamtube (QMS) model [12], which nested the double-multiple streamtube (DMS) model [13], was used as the flow field model. The flow field was divided into 20 in the height direction and was divided into 180 in the perpendicular direction to the main stream. In the direction of the main stream, the flow is divided into four portions (QMS) or two portions (DMS), depending on the number intersecting the blades. A decrease in rotor output power caused by blade twist was simulated by considering the reduction of the swept area $A$ and the effective pitch angle $\theta$ [14]. In the present study, the effective pitch angle caused by blade twist is defined by Equation (8):

$$
\theta=\tan ^{-1} \frac{z \sin \eta}{r}
$$

where $r$ is the local radius from a base point in a blade cross section to the rotor rotational axis and $z$ is the local height of the base point from the equator plane.

The BEM-based prediction needs aerodynamic data of the blade section (NACA 0018). The two-dimensional aerodynamic data of lift and drag coefficients $\left(C_{\mathrm{L} 2 \mathrm{~d}}, C_{\mathrm{D} 2 \mathrm{~d}}\right)$ were prepared from the combination of the data reported by Sheldahl and Klimas [15] and the data proposed by Kumar et al. [16] for a low Reynolds number. The data on moment coefficient $\left(C_{\mathrm{M} 2 \mathrm{~d}}\right)$ around the quarter point of a blade chord was obtained from two-dimensional computational fluid dynamics (CFD) analysis [17] previously carried out at Tottori University. In order to consider the effects of induced drag, the data correction method reported by Murata et al. [18] was used in this study. The dynamic stall model proposed by Paraschivoiu based on the modified Gormont model [12] was also introduced in the prediction.

In the present prediction, from the experiences based on some previous experiments and CFD analyses $[7,13]$, the upstream speed of the double-blade structure rotor is corrected by Equation (9):

$$
V_{\infty}^{c}=V_{\infty}\left\{1-F_{f}\left(0.1028 \frac{r_{2}}{r_{1}}-0.0151\right) \sigma_{\text {in }}^{\prime}\right\}
$$

where $V_{\infty}$ is the upstream uniform velocity speed and $V_{\infty}{ }^{c}$ is the corrected upstream speed of each streamtube located at an arbitrary local height. $r_{1}$ and $r_{2}$ are the local radius of outer rotor and the 
local radius of inner rotor, respectively, at an arbitrary height. The values of $r_{1}$ and $r_{2}$ vary depending on the height after the blade twist. $\sigma^{\prime}$ in is the local solidity of the inner rotor and is defined by $B c_{2} / r_{2}$. $B$ is the number of blades and $c_{2}$ is the blade chord length of the inner rotor corresponding to the local radius $r_{2} . F_{f}$ is introduced as a fitting parameter that does not depend on the local height.

In this study, the tuning of in-house software for the performance prediction was conducted by fitting of the torque curve predicted by the above-mentioned BEM method to the experimental torque curve obtained from the previous generation experiments of a 3 m-diameter BWT with four circular blades (ACBBWT) [19]. In that process, the experimental power performance was converted to the torque performance by assuming the generator loss of $20 \%$. Figure 10 is the result of the best fitting, in which the value of the fitting parameter $F_{f}$ is 0.4 .

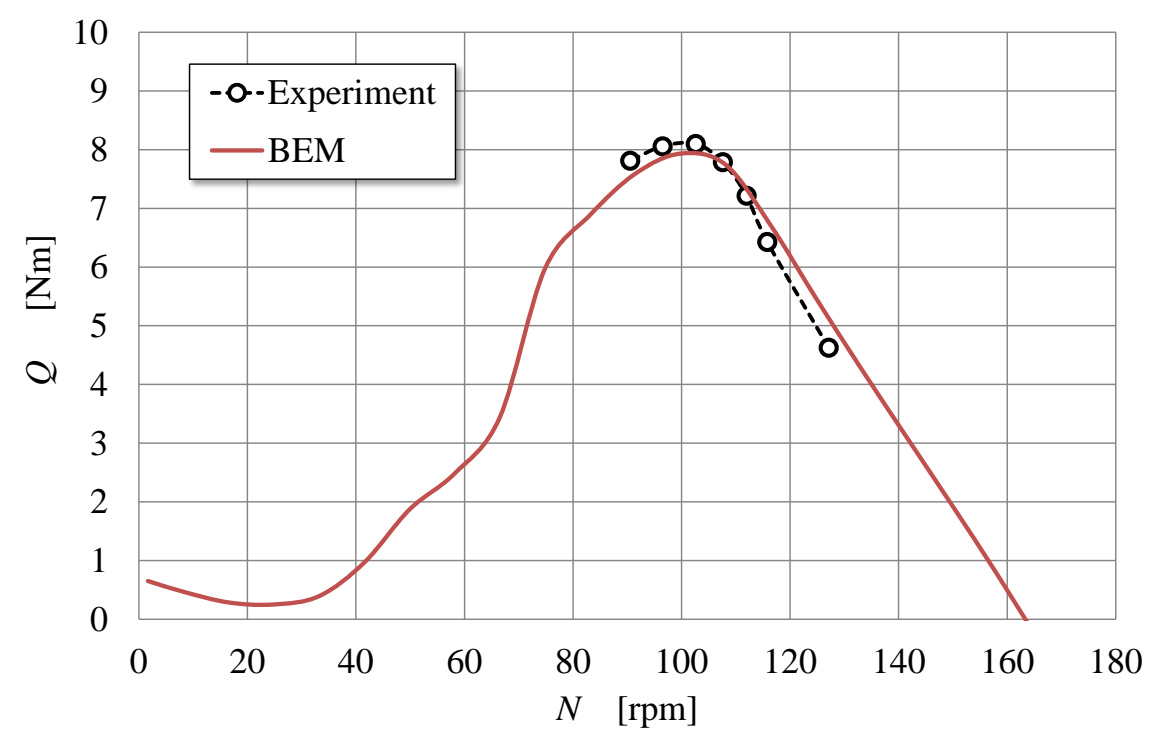

Figure 10. Fitting of the torque curve calculated by the blade element momentum (BEM) theory to the experimental data of a $3 \mathrm{~m}$ BWT with four circular blades [15]. The averaged wind speed in the experiment with using an outdoor blower $(\varphi 3 \mathrm{~m})$ was $5.4 \mathrm{~m} / \mathrm{s}$, which was measured by a cup anemometer placed at $3 \mathrm{~m}$ upstream from the turbine rotor center.

The prediction of torque curves of $7 \mathrm{~m}$-diameter BWT without OCS, i.e., in the no-twist condition, is illustrated at intervals of $1 \mathrm{~m} / \mathrm{s}$ from $1 \mathrm{~m} / \mathrm{s}$ to $10 \mathrm{~m} / \mathrm{s}$ in Figure 11 . The broken lines are the experimentally obtained torque characteristics of the coreless generator (see Figure 9) combined with the increasing gear (3:1) and they depend on the value of external resistance ( $24 \Omega-300 \Omega$ ). Since the horizontal axis of Figure 11 is indicated by the rotational speed of the turbine rotor, please note that the generator speed increased by the gear is three times as high as the speed indicated by the horizontal axis. A line in red indicates the maximum output conditions at each wind speed. When wind speed is $7 \mathrm{~m} / \mathrm{s}$ or less, optimum control can be theoretically realized. That is, the output resistance of the generator is controlled so as to reconcile the intersection (operating point) of a rotor torque curve and a generator torque curve to a point on the maximum power curve by changing the pulse width of the output signals of the generator. However, in the case of Figure 11, the operating point cannot be on the maximum power curve when the wind speed is $8 \mathrm{~m} / \mathrm{s}$ or more because the maximum current, or torque, of the generator is limited by the allowed smallest external (or output) resistance of $24 \Omega$. Therefore, in that case, the pulse width modulation (PWM) is performed to get the operating point positioned on the generator torque curve of $24 \Omega$ and to be less than the rated rotor speed of $110 \mathrm{rpm}$. In general, at the resultant operating point, the output power decreases and the rotational speed increases. 


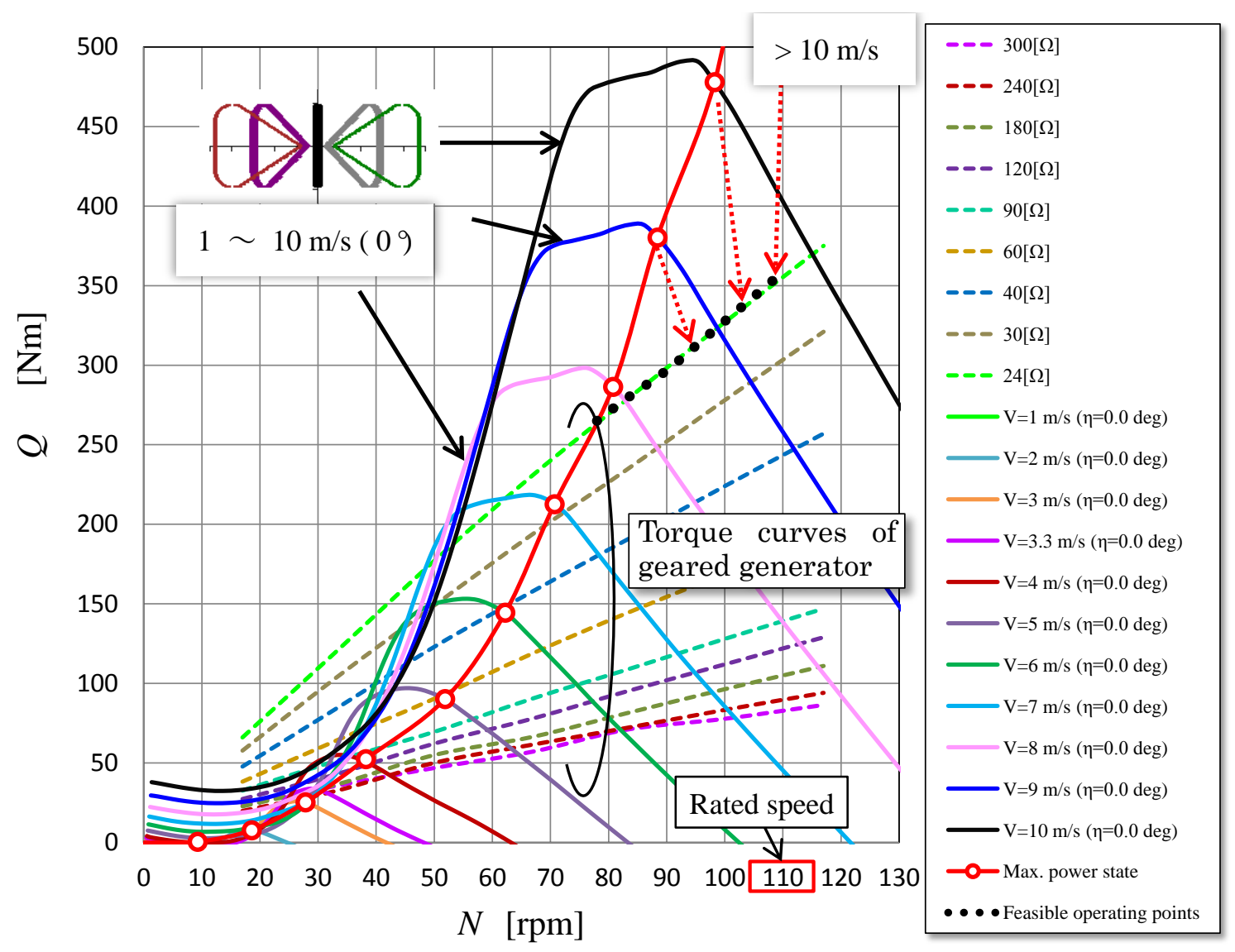

Figure 11. Torque performance of the BWT without the OCS and geared generator.

Figure 12 illustrates the predicted torque characteristics of the BWT equipped with the modified OCS. The rotor torque curves when the wind speed is $8 \mathrm{~m} / \mathrm{s}$ or lower are almost the same as those in Figure 11, because the blades do not twist at all or the twist angle is very small even if they twist. When the wind speed is $9 \mathrm{~m} / \mathrm{s}$ or higher, the decrease of maximum torque is predicted because of the blade twist. According to the prediction, in the case of wind speed of $12.8 \mathrm{~m} / \mathrm{s}$, the twist angle becomes 12 degrees and the turbine rotor rotates in a condition close to the rated speed. If wind speed is higher than the rated wind speed (about $13 \mathrm{~m} / \mathrm{s}$ ), as in the case of $17 \mathrm{~m} / \mathrm{s}$ shown in Figure 12, the rotor torque curve cannot intersect with the generator torque curve of $24 \Omega$ of the control target, because the blades are inclined to a large twist angle $\left(15.5^{\circ}\right.$ at $\left.17 \mathrm{~m} / \mathrm{s}\right)$ and the maximum rotor torque is greatly reduced. That state means that the turbine rotor cannot rotate the generator due to large aerodynamic resistance and the rotor rotational speed must be reduced. However, if the strong wind over the rated wind speed continues to blow, the turbine rotor is accelerated again after slowing down and obtaining a small twist angle. An increase and decrease of rotor rotation near the rated rotor speed is predicted and an almost constant output power could be continued.

For reference, the non-dimensional torque and power curves of the BWT are shown in Figures A1 and A2 of Appendix A. The maximum power coefficient of 0.415 is predicted in the case of $8 \mathrm{~m} / \mathrm{s}$ without twist due to less tip loss and arm drag as the features of the BWT. 


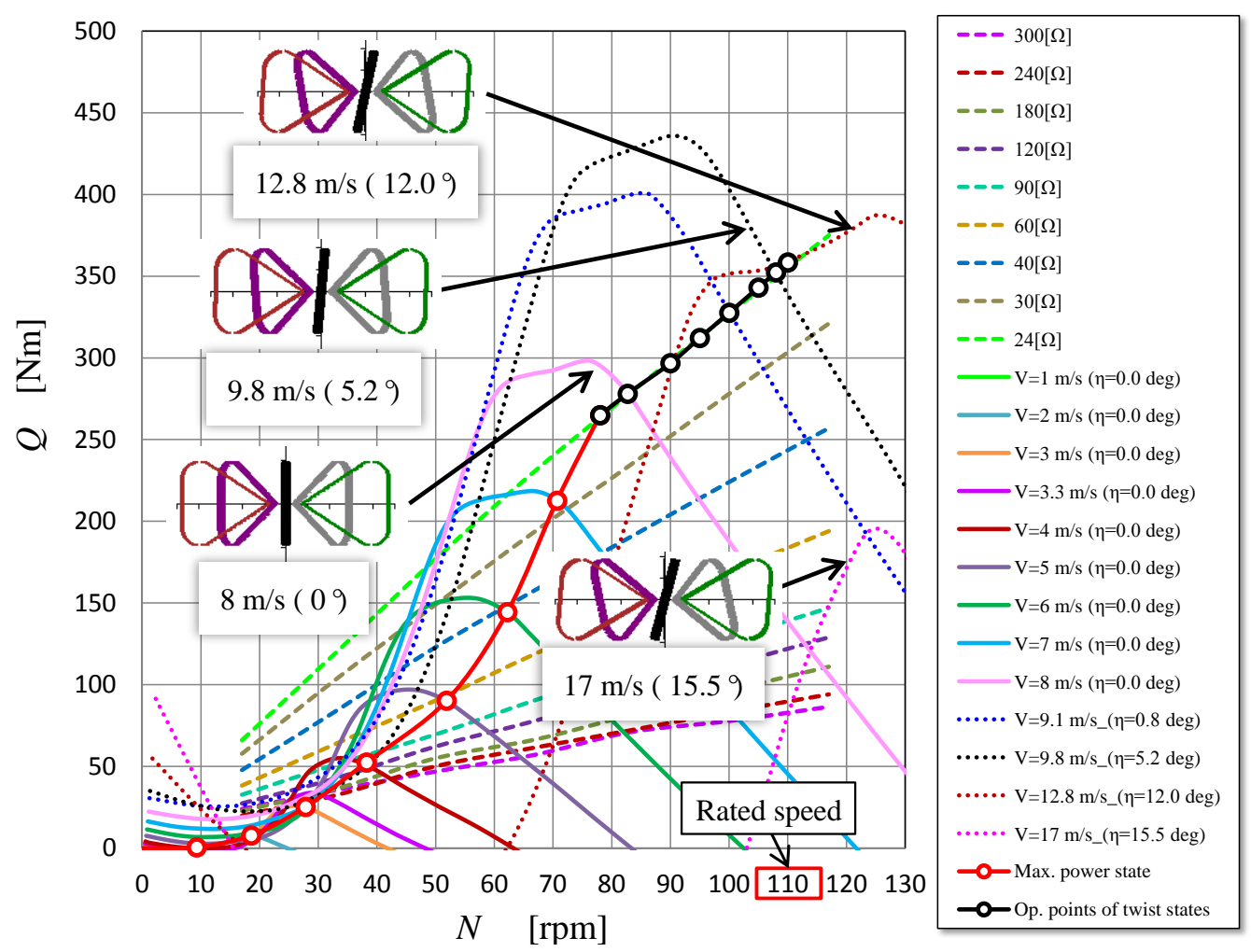

Figure 12. Torque performance of the BWT with the OCS and geared generator.

Comparisons between the power curves of the BWT with and without the OCS is shown in Figure 13, which are calculated based on the torque curves shown in Figures 11 and 12. In the case without the OCS, the use of a virtual generator is assumed until the cutout wind speed of $12 \mathrm{~m} / \mathrm{s}$ (the rated electric power: $6674 \mathrm{~W}$ ) with a control tracking the maximum power conditions denoted by the line in red shown in Figure 11. In Figure 13, the electric power is calculated by considering the total efficiency (e.g., $150 \mathrm{rpm}, 60 \Omega: 63.6 \% ; 351 \mathrm{rpm}, 24 \Omega: 76.4 \%$ ) of the generator and the speed-increasing gear based on the results of preliminary experiments. In the case of the BWT with the OCS, the maximum electric power (the rated electric power: $3145 \mathrm{~W}$ ) is much smaller than the case of the BWT without the OCS. However, the cutout is not defined theoretically, although the power curve of the BWT with the OCS is not illustrated at the wind condition of $31 \mathrm{~m} / \mathrm{s}$ or higher in Figure 13.

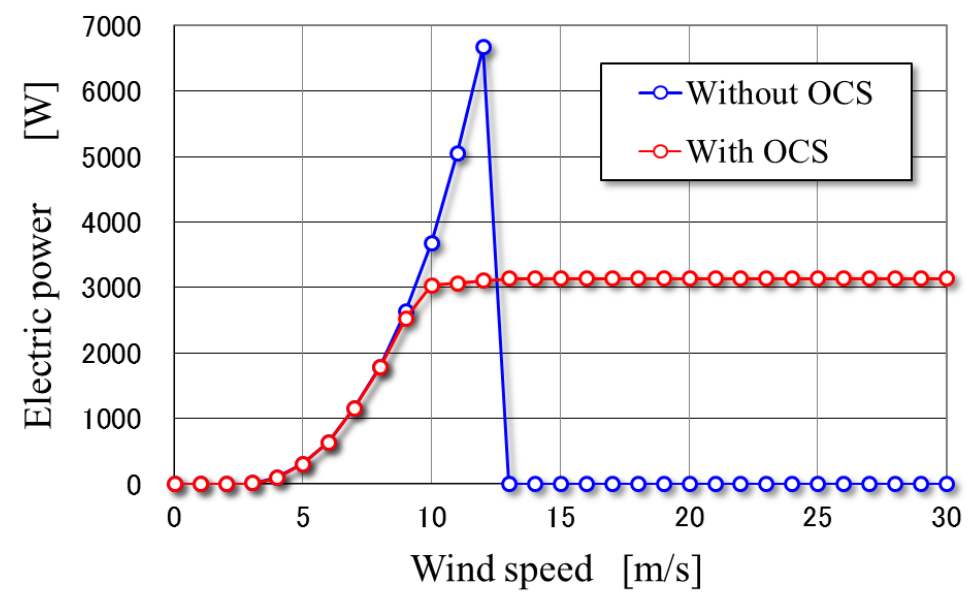

Figure 13. Comparison of power curves between the BWTs with and without the OCS. 
The predictions of the annual energy production (AEP) based on the power curves in Figure 13 are shown in Figure 14, in which the Rayleigh distribution [20] was used to represent the wind speed probability density. Figure 14 shows that there is little difference in AEP between the BWT without the OCS and the BWT with the OCS at the annual average wind speed of $7 \mathrm{~m} / \mathrm{s}$ or lower. According to the prediction, the AEP of the BWT with the OCS amounts to $5791 \mathrm{kWh}$ at the site where the annual average wind speed is expected to be $5 \mathrm{~m} / \mathrm{s}$.

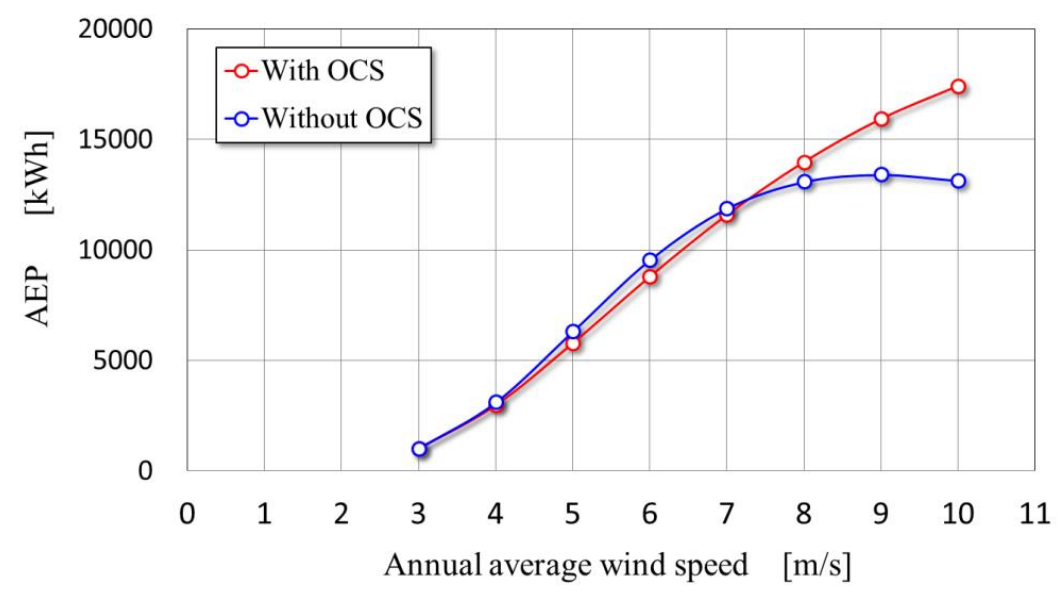

Figure 14. Comparison of annual energy production (AEP) between the BWTs with and without the OCS.

Although the difference of AEP between both turbines with and without the OCS is little in the case of low annual average wind speed, as mentioned above, the wind power system without the OCS will be expensive because it needs a generator of large capacity and, therefore, other electrical devices of high performance. On the other hand, the total cost of the wind-power system with the OCS will be reduced because the capacities of the electrical devices could be small, although the manufacturing cost of the OCS is added.

In addition, if the turbine rotor is equipped with a simple stopper to hold the rotor for maintenance, the system with the OCS needs no other mechanical brake such as a disk brake. The capacity factor of the BWT with the OCS is improved, as understood from Figure 13. The effects will be produced at the high wind potential sites as shown in Figure 14. The wind-power system is controlled electrically by a controller regardless of the presence of the OCS. Although the wind-power system without the OCS might reach an over-speed condition in the case of loss of electrical control, the system with the mechanical OCS could prevent over-speed even when some electrical system problem occurs.

\subsection{Power-Generation Control System}

In the project, at first, the wind-power generation system was designed for grid connection, in which the generated electricity was supposed to be supplied to the grid through a power conditioning system (PCS). The control of the wind turbine was planned to be carried out by the function of maximum power point tracking (MPPT) of the PCS. However, in resolving the difficulty of getting permission early for grid connection because of the first development of a wind turbine with a special shape, the system was altered to use both the independent operation of the PCS and power-generation controller with a data table in order to give priority to the investigation of the generation performance of the BWT equipped with the OCS.

Figure 15 shows the inside of the first control console planned in the project. The PCS (Diamond Electric Mfg. Co., Ltd., Osaka, Japan, DPC-45B, $4.5 \mathrm{~kW}$ ) is an inside-installation type for the photovoltaic power-generation system. In the preceding stage, the controller No. 1 (SKY Electronics Co., Ltd., Yonago, Japan, CL302) is installed. The resistor No. $1(2 \mathrm{~kW} / 10 \Omega \times 3)$ is used to consume the generated electric power in case of emergency stop. Apart from this, another resistor $(1 \mathrm{~kW} / 2.2 \Omega \times 1)$ 
is equipped for functioning as a short-circuit brake to hold the rotor. The data logger system (sampling interval: about $1.5 \mathrm{~s}$ ) records generator conditions (voltage, current, electric power, and rotational speed), wind conditions (wind speed and direction), status of the PCS, and so forth, and outputs those data to an SD memory card.

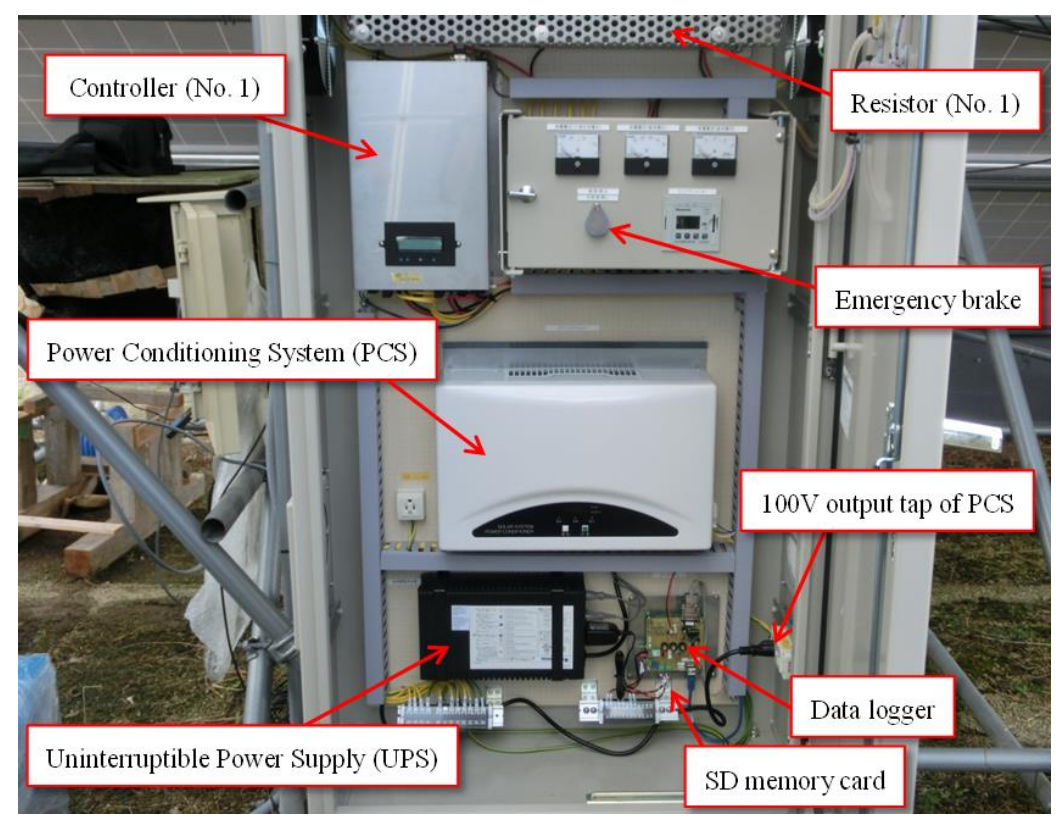

Figure 15. Control console for the wind turbine before adding the second controller and resistor.

Figure 16 shows the generation controller No. 2 (SKY Electronics Co., Ltd., Yonago, Japan, CL302) and the resistor No. $2(1 \mathrm{~kW} / 10 \Omega \times 2)$, which were installed on the inner door panel of the control console after the alteration of the first plan.

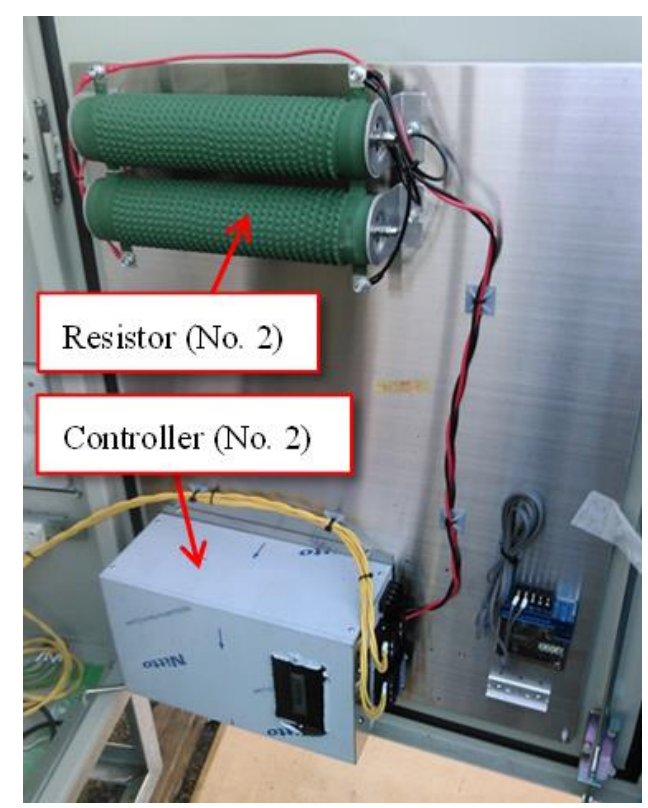

Figure 16. Second controller and resistor installed on the inner door panel of the control console. 
The schematic diagram of the power-generation control system after the plan alteration is illustrated in Figure 17. When the electric power is $2 \mathrm{~kW}$ or less, the controller No. 1 controls the input current to the resistor No. 1 by PWM referring to a data table of target electric power against the generated voltage. Most of the generated electricity is consumed by the resistor No. 1. When the generated power exceeds $2 \mathrm{~kW}$, the excess electric power passing through the PCS of independent operation is consumed at resistor No. 2, in which the controller No. 2 is used to track the target condition.

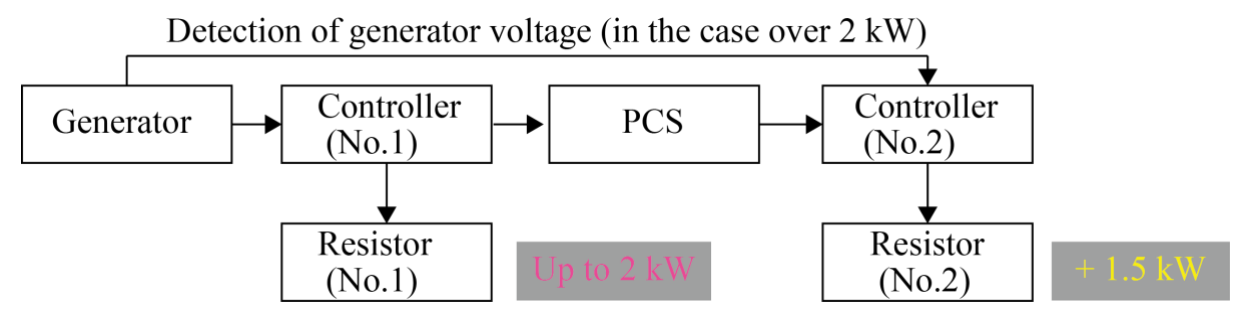

Figure 17. Schematic diagram illustrating the power-generation control.

Figure 18a shows the target electric power against the generator voltage. As shown in Figure 18a, the power-generation control system can deal with the maximum electric power of $3.5 \mathrm{~kW}$. When the rotor rotational speed is low, the control system makes almost unloaded state, or non-electric current state, to improve the starting characteristics of the wind turbine rotor. The graph near the generation start point is magnified in Figure $18 \mathrm{~b}$. The standby power $(20 \mathrm{~W})$ is supplied to the PCS when the generator voltage becomes more than $96 \mathrm{~V}$, which corresponds to the rotor rotational speed of about $34 \mathrm{rpm}$. When the voltage reaches $105 \mathrm{~V}$, the electric current starts to flow into the resistor No.1. Since the PCS is originally for grid connection, the independent mode of the PCS cannot continue by default. Therefore, a part of the control program of the PCS was altered so as to continue the independent mode by the manufacturer of the PCS.

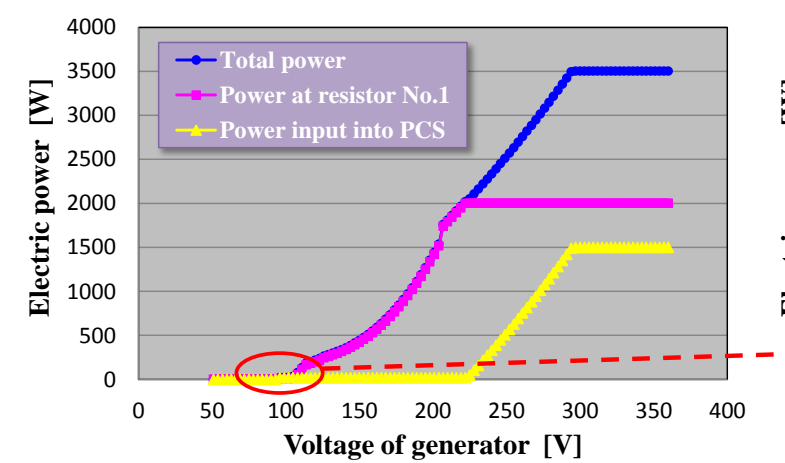

(a)

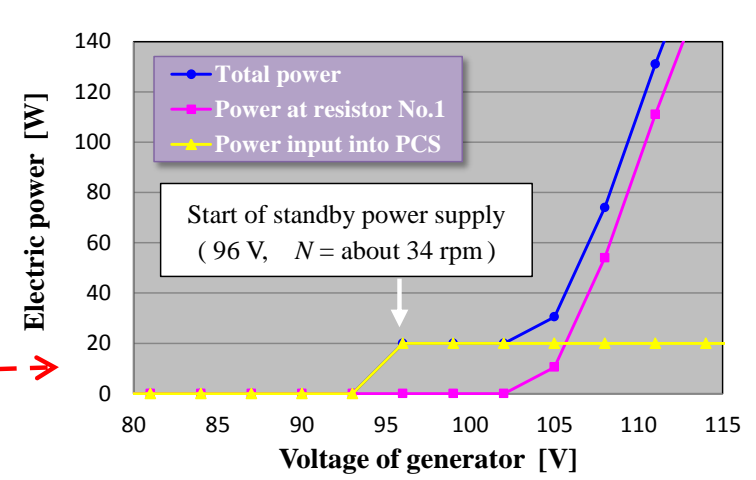

(b)

Figure 18. Target values in the power-generation control. Lines in pink show the electric power consumed at resistor No.1 and lines in yellow show the electric power input into the PCS including the power consumed at resistor No.2. Lines in blue are the total power generated: (a) whole graph;

(b) magnified graph around the generation start point.

\subsection{Site of Experiments}

The prototype of the BWT has been installed in one corner of the site of Hokuei Takachiho Solar Power Plant (Enatex Co., Ltd., Kurayoshi, Japan) [21], which is located in the Middle District of Tottori Prefecture in Japan. A photograph and a drawing of the site are shown in Figure 19. The experimental turbine is located close to solar panels and there is an upsurge of land which is covered by leafy trees 
on the south side of the turbine. In spite of the bad conditions for wind power generation, this site was selected because of the easiness of both managing the wind turbine and implementing the forcible rotation experiments that should be carried out in low wind-speed conditions. The wind turbine can rotate well only when the wind blows from the west or the south-west. Therefore, a two-dimensional ultrasonic anemometer (CLIMATEC, Inc., Tokyo, Japan, CYG-85000) was installed at $20 \mathrm{~m}$ in the western direction from the wind turbine in order to monitor the wind speed and wind direction. The height of the anemometer is almost the same as the center of the wind turbine rotor.

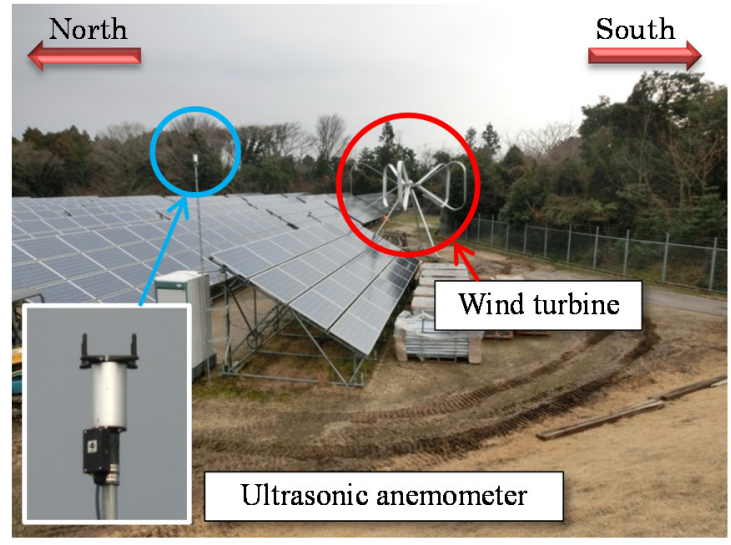

(a)

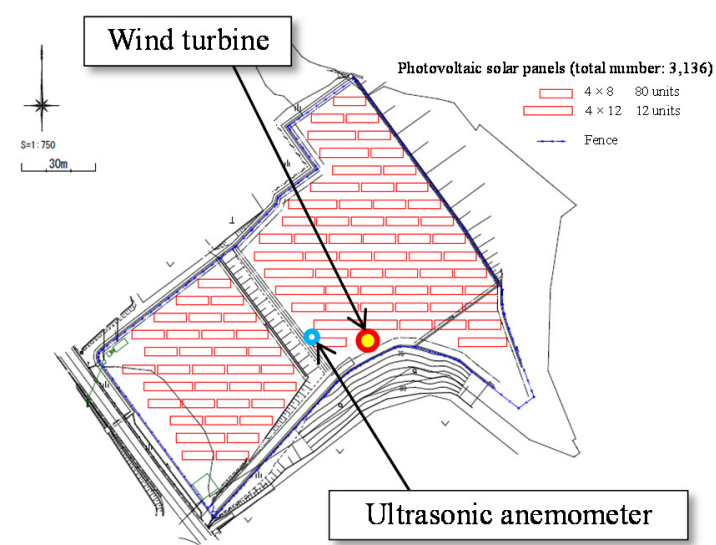

(b)

Figure 19. Experimental site: (a) photograph taken from the west of the wind turbine, including an embedded photo showing an ultrasonic anemometer used for monitoring the wind conditions; (b) drawing of the solar power plant where the experimental wind turbine was installed. The two-dimensional ultrasonic anemometer was installed at $20 \mathrm{~m}$ in the western direction from the wind turbine.

\section{Experimental Results}

In this section, the results of the fourth experiment series of forcible rotations are described in Section 3.1 and the results of the power-generation experiments by natural wind are shown in Section 3.2. The forcible rotational experiments were conducted by using a geared induction motor (Tsubakimoto Chain Co., Osaka, Japan, GMTE370-50L15BFI, $3.7 \mathrm{~kW}, 4$ poles, 1:15) instead of the generator in low wind-speed conditions. The power-generation experiments had been carried out by applying adjustments or alterations to the system repeatedly since October 2017. In this paper, the results of the experimental observation on 1 March 2018 are reported, where the power-generation system is applied with the alteration explained in Figures 17 and 18.

\subsection{Forcible Rotation Using Induction Motor}

The photographs taken in the fourth experiment-series of forcible rotation carried out with the guide grooves of final design depicted in Figure 7 are shown in Figure 20. Figure 20a concerns the stationary state $\left(\eta=0^{\circ}\right)$ of a blade and Figure $20 \mathrm{~b}$ concerns the maximum twist state $\left(\eta=\right.$ about $\left.12^{\circ}\right)$ of the blade during high rotational speed. Those two images were obtained from a digital camera placed on the top of the rotor hub. The photographs about the synchronizing disk positioned at the center of the OCS shown in Figure 20c,d were obtained at the corresponding times to Figure 20a,b, respectively. The central angle between the line drawn from a pivot of a horizontal link to the center of the disk and the vertical base line was changed by $\Delta \xi=\xi_{2}-\xi_{1}=34.2^{\circ}$ during the blade twist. 


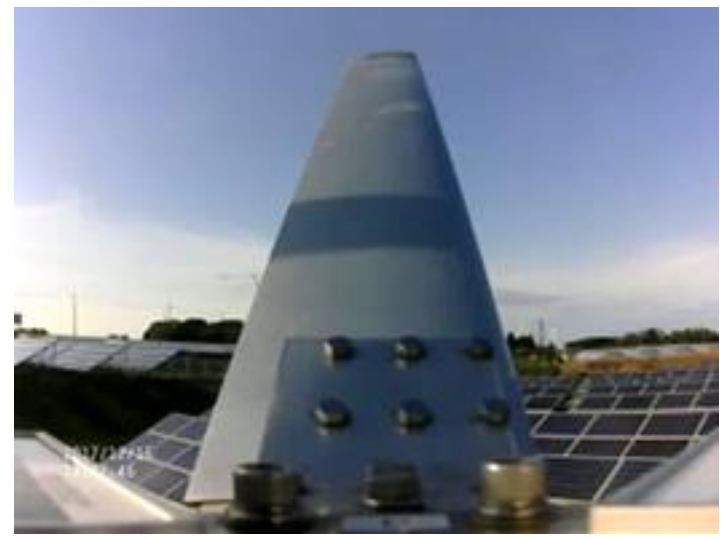

(a)

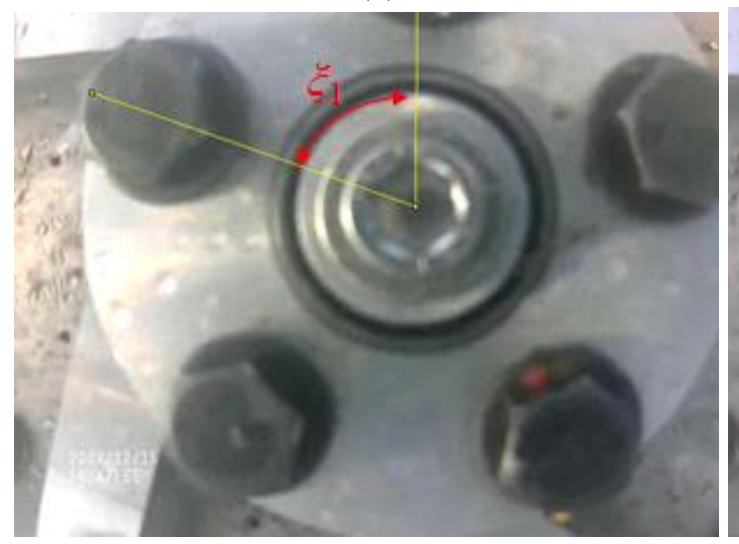

(c)

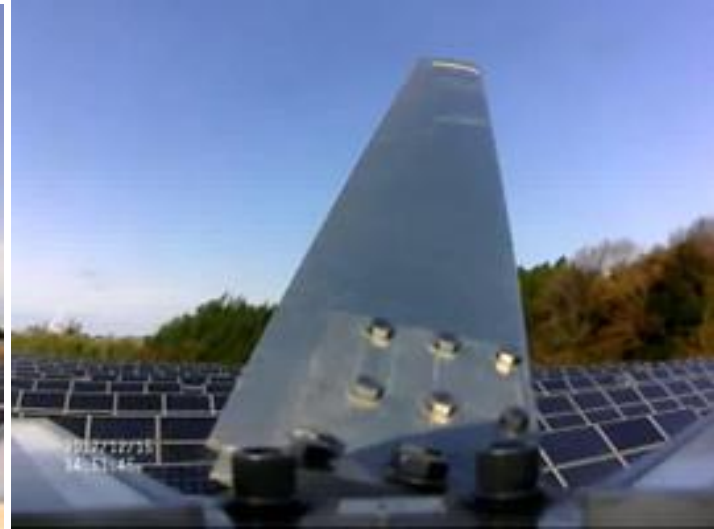

(b)

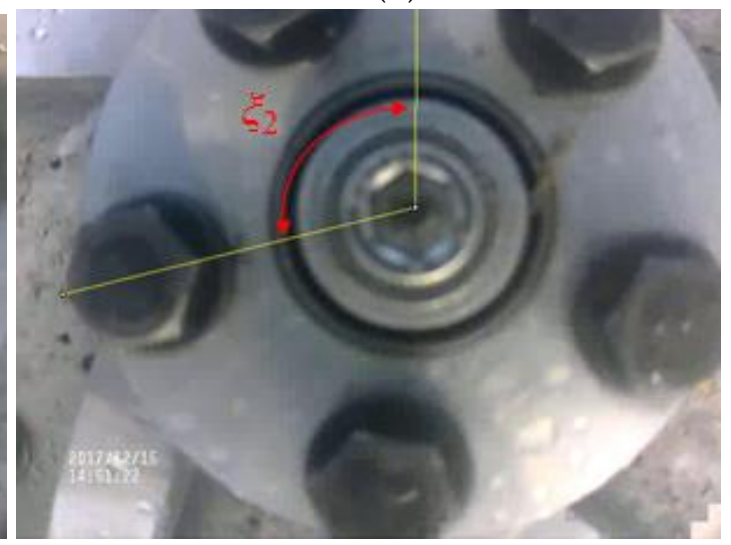

(d)

Figure 20. Photographs taken by the cameras installed on the hub of wind turbine during the fourth experiment series of forcible rotations by an induction motor, where the final design of guide grooves was used. The upper figures show the conditions of a looped blade; (a) stationary state; (b) maximum twist state $\left(\eta=\right.$ about $\left.12^{\circ}\right)$. The bottom figures show the synchronizing disk; (c) stationary state $\left(\Delta \xi=0^{\circ}\right)$; (d) maximum twist state $\left(\Delta \xi=34.2^{\circ}\right)$.

Figure 21 is an example of the results obtained in the fourth experiment series of forcible rotations regarding the time-series variation of the turbine rotor speed $N$, the blade-axis displacement $\Delta x$, and the twist angle $\eta$. The turbine rotor speed was gradually varied by manually increasing the frequency of the inverter controlling the induction motor. The rotational speed of the turbine rotor was measured by an optical detector (Ono Sokki Co., Ltd., Yokohama, Japan, LG-930), which detected the reflected light from the 36 reflection stickers on the bottom face of the rotor hub. At first, the change $\Delta \xi$ in the central angle of the synchronizing disk was obtained at intervals of about $5 \mathrm{~s}$ from the video analysis. Then, the displacement $\Delta x$ was calculated by Equations (1)-(3). The twist angle $\eta$ corresponding to each value of $\Delta x$ was calculated based on the shape of a guide groove. In Figure 21, the blade-axis displacement $\Delta x$ abruptly increases past the time of $185 \mathrm{~s}$, at which the turbine rotor speed $N$ reaches the maximum of $101.5 \mathrm{rpm}$, and, simultaneously, the twist angle $\eta$ increases up to about $12^{\circ}$. Soon afterwards, the motor becomes over-loaded and cannot be controlled by the inverter. Therefore, the rotor speed decreases. After a while, past $255 \mathrm{~s}(\mathrm{~N}$ : around $75 \mathrm{rpm})$, the displacement and the twist angle restore the original states. 


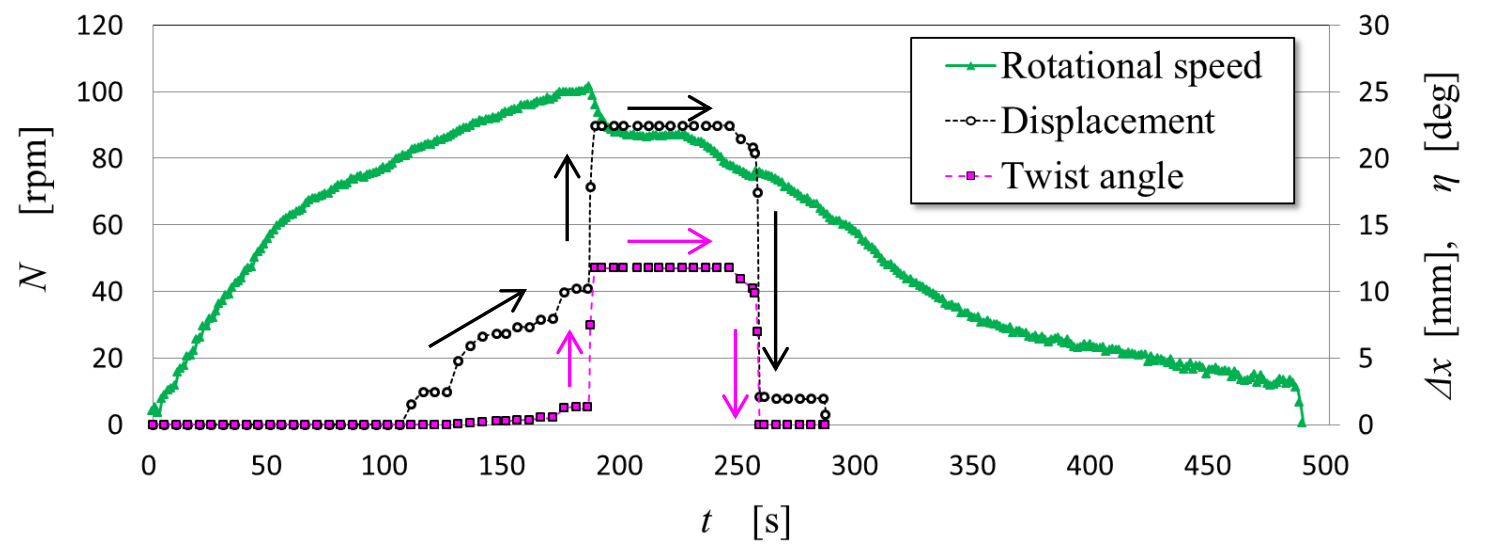

Figure 21. Variations in rotational speed $N$, displacement $\Delta x$ of a blade axis, and twist angle $\eta$ in the forcible experiments using the final design of the guide grooves.

Figure 22 shows the relation between the twist angle $\eta$ and the turbine rotor speed $N$, which is obtained from the experimental data shown in Figure 21. A curve in black depicted in Figure 22 is the prediction shown in Figure 8. Despite an abrupt increase in twist angle around the rotor speed of $110 \mathrm{rpm}$ being predicted, as deduced by the results of the fourth forcible rotation experiments, the twist angle increased more abruptly and at smaller rotor speed. Ideally, it is desirable that the twist angle decreases quickly along the predicted curve after the decrease in rotor speed by blade twist. However, large hysteresis shown in Figure 22 was observed. That is probably attributed to the friction between the guide grooves and the pin that moves along them. Nevertheless, the results shown in Figure 22 have been greatly improved compared to the results of the previous forcible rotation experiments (see Figure 5b).

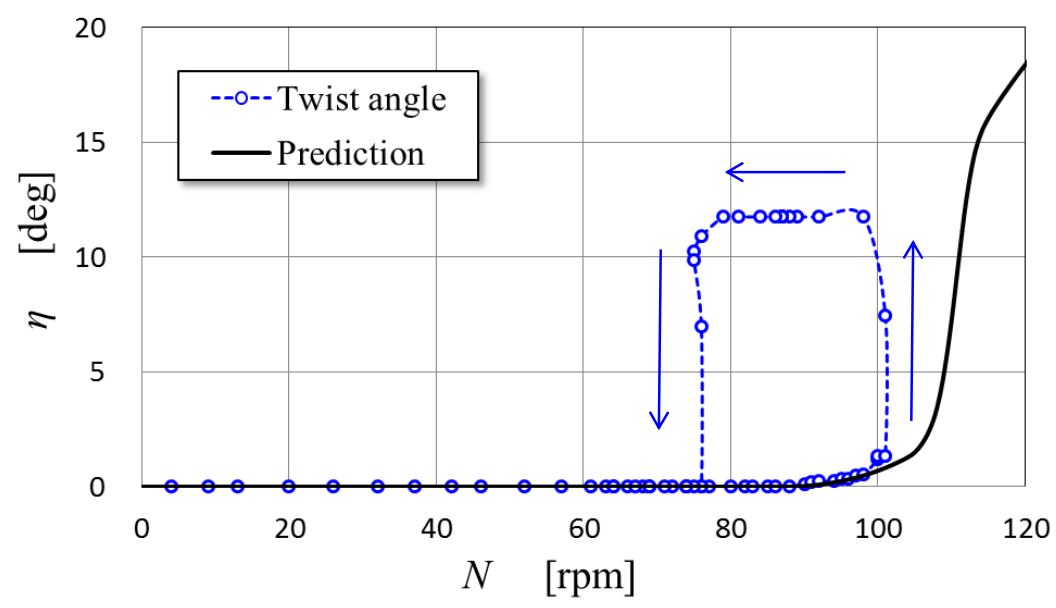

Figure 22. Relationship observed between the twist angle $\eta$ and the rotor rotational speed $N$ in the forcible experiments using the final design of the guide grooves. The line in black is the prediction based on both the centrifugal force and the radial force $F_{t w}$ observed in the previous experiments.

\subsection{Experiments of Power Generation}

Relatively strong wind blew through the day on 1 March 2018. On that day, the averaged wind speed throughout the day was $3.8 \mathrm{~m} / \mathrm{s}$ and the averaged wind direction was $227.8^{\circ}$ of a south-western wind. During $15 \mathrm{~h}$ between 5:00 and 20:00, the wind direction was stable west-south-west (average wind direction of $15 \mathrm{~h}: 239.9^{\circ}$ ) and the wind speed was rather strong (average wind speed of $15 \mathrm{~h}: 4.6 \mathrm{~m} / \mathrm{s})$. Therefore, the experimental wind turbine rotated well and substantial electric power 
generation was observed during the $15 \mathrm{~h}$. Figure 23 shows the data of wind speed, wind direction, generator voltage, electric power, and rotor rotational speed, observed on 1 March 2018. The rotor rotational speed shown in Figure 23 was obtained from the data of variation in the generator voltage due to the malfunction of the optical detector. The difference between the rotor rotational speeds obtained from the generator voltage variation and the optical detector is about $1.7 \mathrm{rpm}$ at the root mean square (RMS) value, according to the comparison conducted in advance. However, when the rotor rotation speed is less than $11 \mathrm{rpm}$, it cannot be obtained, i.e., measured, from the generator voltage variation. The graphs shown in Figure 23 are represented at the intervals of $150 \mathrm{~s}$ by the data, which are the 5-min moving average of the recorded data with sampling times of about $1.5 \mathrm{~s}$. During a time period between 6:55:50 and 7:25:58, the wind turbine revolved very slowly and no electric power was generated in spite of high wind speed conditions. This incident was caused by the automatic operation of the emergency brake, which was triggered by some electrical noise that might be attributed to the matching problem between the generation controllers and the peripheral devices such as the PCS. The thresholds designating the start of the emergency brake were adjusted afterwards.

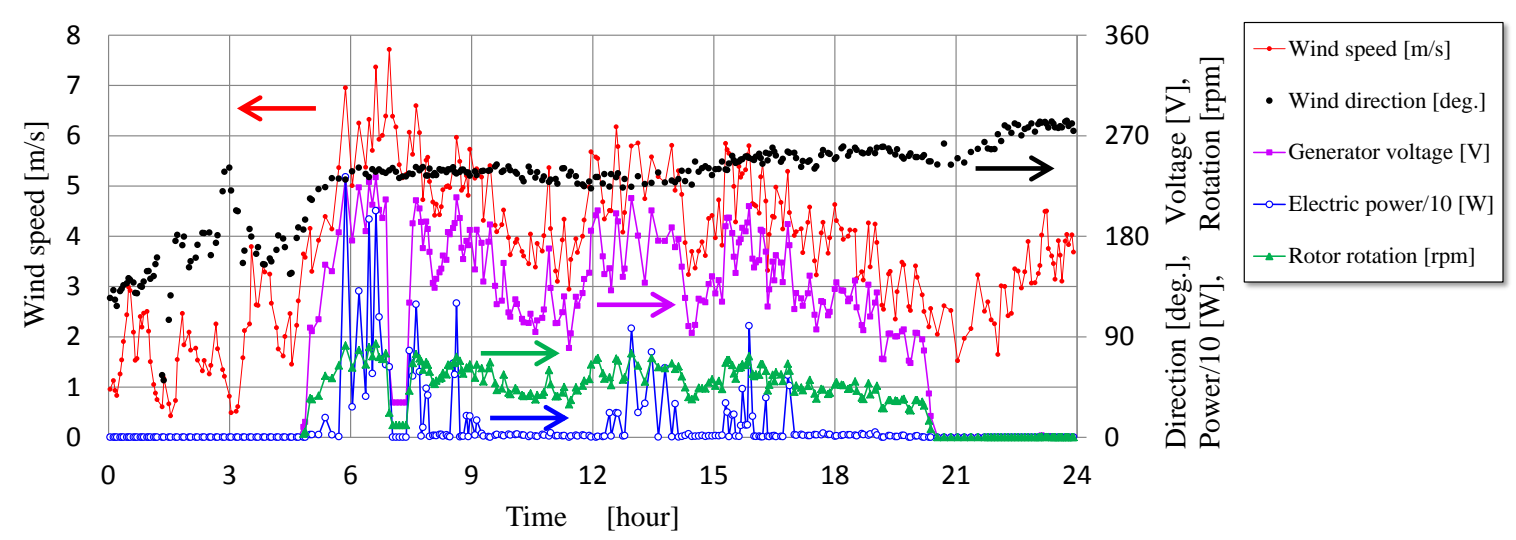

Figure 23. Data obtained on 1 March 2018 (5-min averaged data are represented at intervals of $150 \mathrm{~s}$ ).

Figure 24 shows the relations between the 5-min averages of wind speed and electric power, which are obtained from the data in the time period between 5:00 and 20:00 in Figure 23. A curve in orange is the prediction of the power curve shown in Figure 13 of the BWT with the OCS.

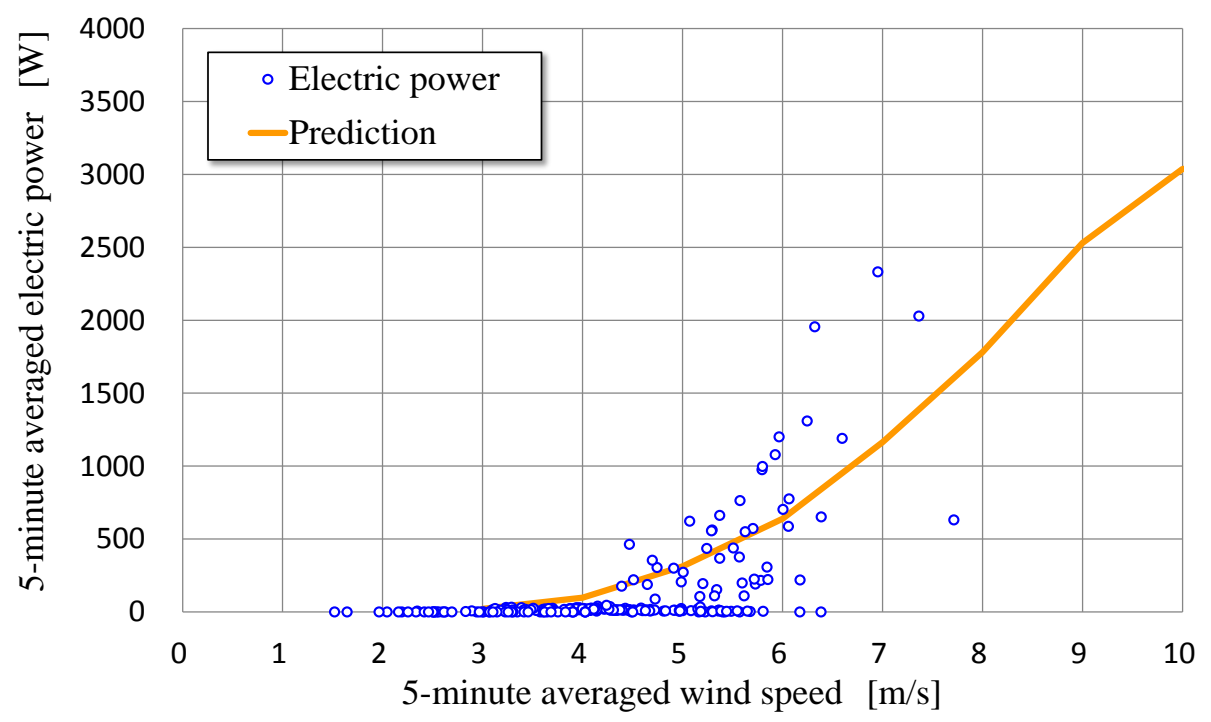

Figure 24. Wind speed dependency of electric power generation, and comparison with the prediction. 
Figure 25, as in Figure 24, represents the relations between the 5-min averages of wind speed and rotor rotational speed. The graph depicted by the symbols in orange shows the predicted rotor rotational speeds corresponding to the operating points shown in Figure 12. Although the observed rotor speeds tend to be a little higher than the predictions, the tendency of both agrees well.

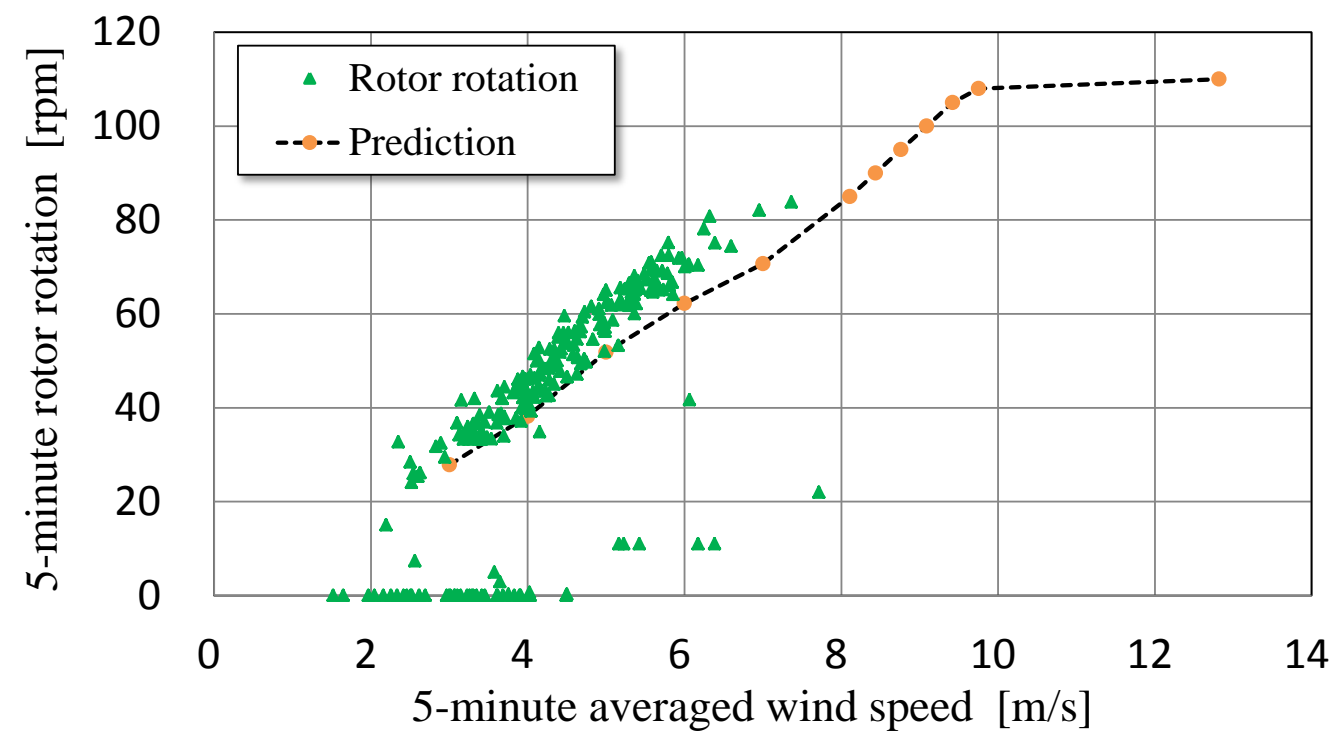

Figure 25. Wind speed dependency of rotor rotational speed, and comparison with the prediction.

Time-series data of rotor rotational speed and wind speed in the time-period from 6:24:38 to 6:25:58 are illustrated in Figure 26, in which the sampling interval is about $1.5 \mathrm{~s}$. At that time period of $80 \mathrm{~s}$, the average wind speed is about $7.3 \mathrm{~m} / \mathrm{s}$ and the average rotor speed is $88.6 \mathrm{rpm}$. The horizontal axis indicates the time that elapsed from 6:24:38. In the data shown in Figure 26, the rotor speed surpassed $100 \mathrm{rpm}$ at $37.4 \mathrm{~s}$ and $38.9 \mathrm{~s}$. The rotational speed of the wind turbine varies relatively slowly with regard to the variation in wind speed. The blade twist state was not confirmed from the video images recorded in the time period shown in Figure 26. However, judging from the aerodynamic noise recorded in the video, it is presumed that the blades might have slanted at a small twist angle during the time period of 30 to $40 \mathrm{~s}$, when the wind turbine revolved at a rotor speed near $100 \mathrm{rpm}$, in Figure 26.

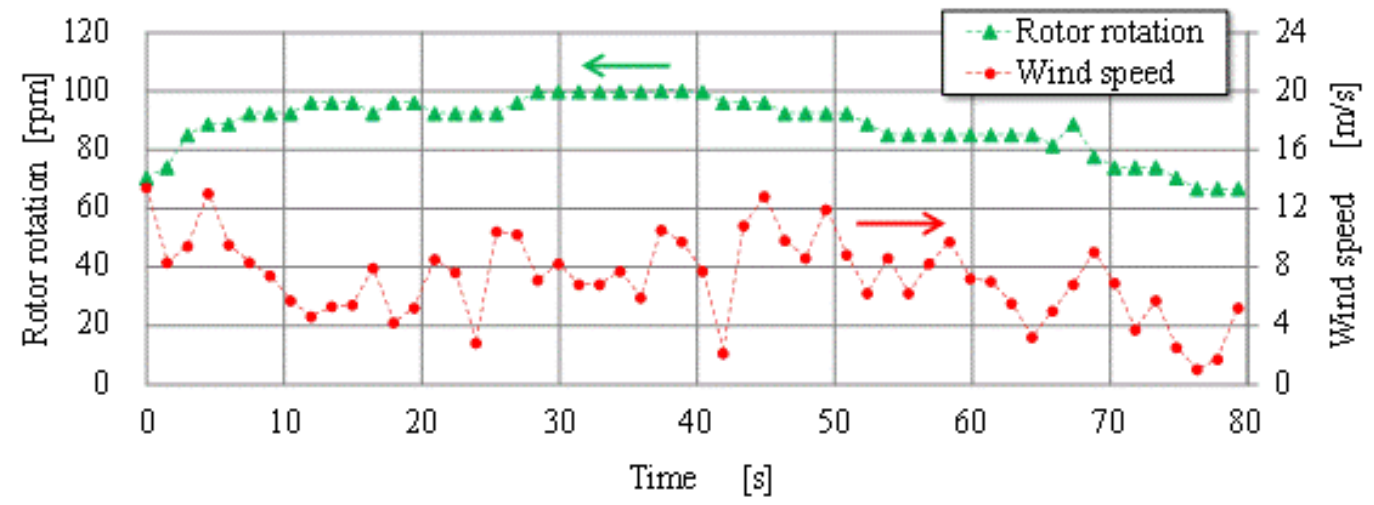

Figure 26. Data of wind speed and rotor rotational speed during the time period from 6:24:38 to 6:25:58 on 1 March 2018.

Figure 27 shows the time-series data of rotor speed and wind speed in the time-period of $40 \mathrm{~s}$ from 6:54:39 to 6:55:19. The maximum wind speed $(18.9 \mathrm{~m} / \mathrm{s})$ in that day was recorded at the elapsed 
time of $15 \mathrm{~s} \mathrm{(6:54:54} \mathrm{at} \mathrm{logger} \mathrm{time)} \mathrm{indicated} \mathrm{by} \mathrm{the} \mathrm{horizontal} \mathrm{axis} \mathrm{in} \mathrm{Figure} \mathrm{27.} \mathrm{After} 1.5 \mathrm{~s}$, at $16.5 \mathrm{~s}$, the rotor rotational speed increased up to the maximum of $107.3 \mathrm{rpm}$ and, from $18 \mathrm{~s}$ to $26.9 \mathrm{~s}$, the blades inclined at a relatively large twist angle.

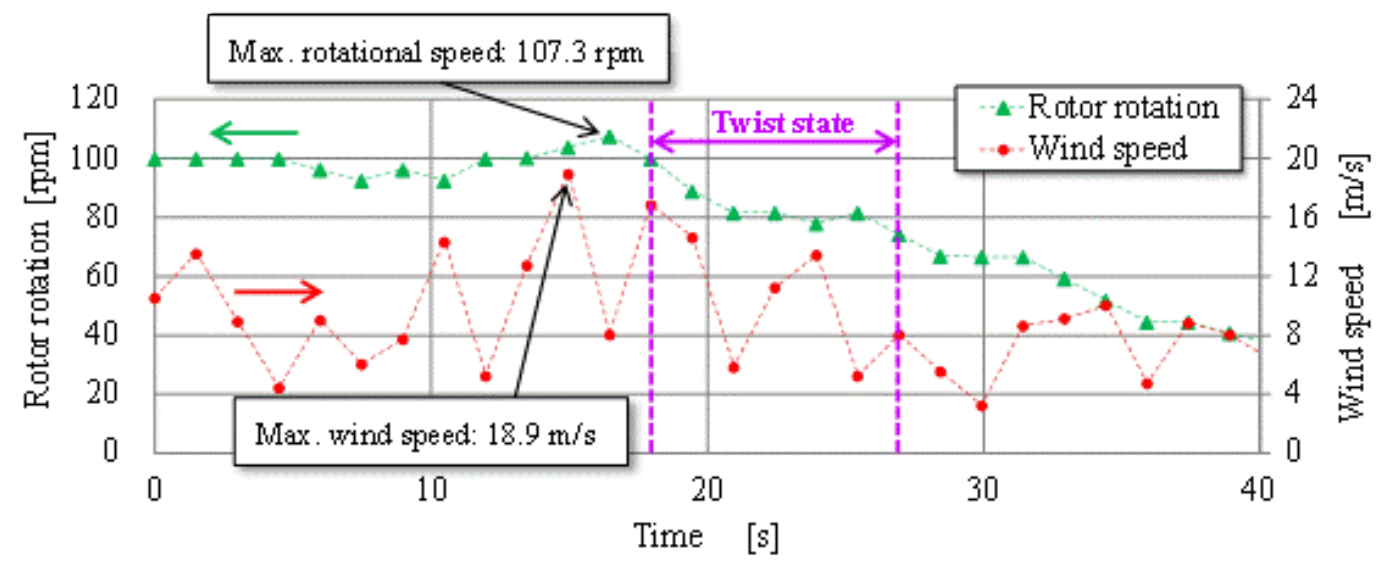

Figure 27. Data of wind speed and rotor rotational speed in the time period from 6:54:39 to 6:55:19 on 1 March 2018.

Two images illustrating (a) no-twist state and (b) twist state of the blades are shown in Figure 28. Those images were recorded by a surveillance camera. Since the time recorded by the camera had a delay of $201 \mathrm{~s}$ compared with the time of the logger, image (a) corresponds to 6:54:51 on the logger time (12 s in Figure 27) and image (b) corresponds to 6:55:00 on the logger time (21 s in Figure 27). The amounts of twist angle and blade-axis displacement of the state shown in Figure 28b were not measured. The decrease (100 to $80 \mathrm{rpm}$ ) in rotor speed observed during the time period of 18 to $25.4 \mathrm{~s}$ in Figure 27 can be probably attributed to the increase in aerodynamic drag caused by the blade twist.

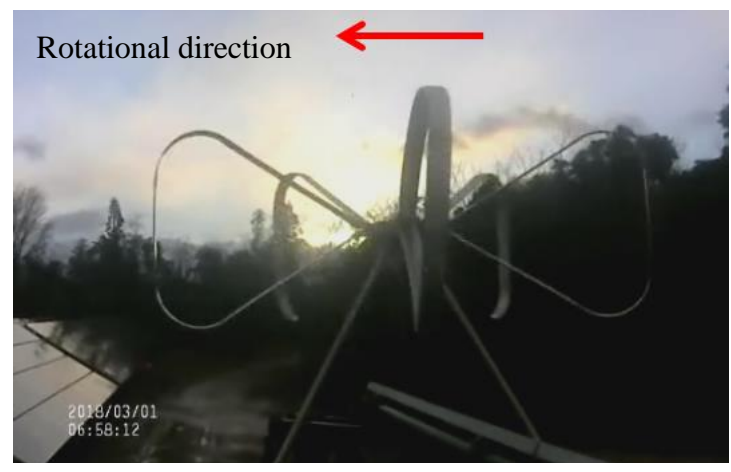

(a)

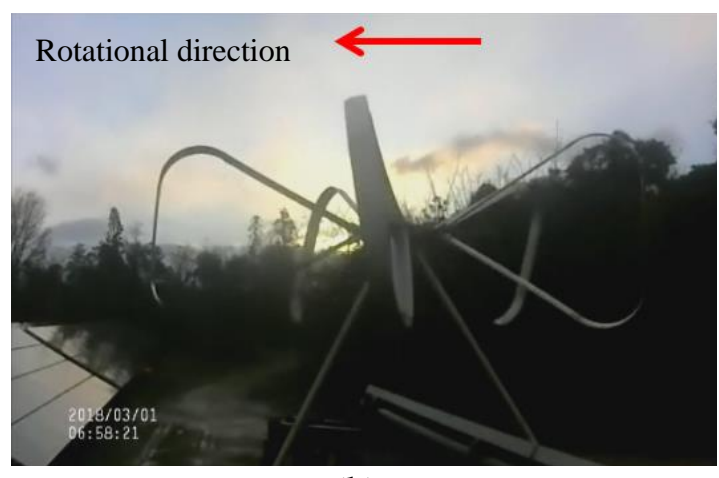

(b)

Figure 28. Twist motion observed in natural strong wind condition on 1 March 2018: (a) no-twist state (6:54:51); (b) twist state (6:55:00).

\section{Discussion}

The maximum rotor speed obtained in the forcible rotation experiments in low wind speed conditions was $101.5 \mathrm{rpm}$, as described in Section 3.1. On the other hand, in the generation experiments shown in Section 3.2, the maximum rotor speed was increased to $107.3 \mathrm{rpm}$. This difference mainly could be caused by the difference in wind speed. However, the possibility of the effects of the uncertain movement caused by friction of the OCS cannot be denied. Nevertheless, it is thought that the relation between the twist angle and rotor speed in the generation experiments under natural wind condition approached the prediction compared with that in the forcible rotational experiments shown 
in Figure 22. In the case of the forcible rotation experiments, more than $60 \mathrm{~s}$ were needed to restore the original state from the twisted state of the blades (see Figure 21). On the other hand, although there was a difference in experimental conditions, the time demanded for the restoration of the twisted blades was about $10 \mathrm{~s}$ in the case of the generation experiments in strong wind conditions (see Figure 27). Unfortunately, since the wind speed became lower after the restoration to the original state, continuous large variation in twist angle was not observed in the experiments. If strong wind conditions were continued, a large variation in twist angle at short intervals around $10 \mathrm{~s}$ would be predicted and power characteristics close to the predicted power curve shown in Figure 13 could be obtained.

According to the relations shown in Figures 24 and 25 regarding the 5-min averages of wind speed, electric power, and rotor rotational speed, moderate agreement between the predictions based on the BEM theory and the experimental data were obtained. However, the amount of experimental data was absolutely insufficient; in particular, data in high wind-speed conditions have not been obtained so far. The experimental wind turbine at the present site cannot be rotated well by wind blowing from various directions, except for the specific directions such as the south-west and west. One of the future works will be to carry out long-term experiments to obtain the performance of the BWT equipped with the OCS by installing it on a flat site with no obstacle.

As described in Section 2.4, a special power-generation control system using two controllers and one PCS that was operated in the independent mode, without the grid connection, was adopted in this study. Since power generation over $2 \mathrm{~kW}$ was observed in Figure 24, the control system worked well without significant problems. The realization of the grid connection of the BWT and the development of the independent power system equipped with an electrical storage device are included in future works. A plan to move the BWT system to the site of the Arid Land Research Center [22] of Tottori University is under consideration. When the plan is realized, some experiments using electrical storage device will be conducted with the aim of applying the BWT as an independent power source to drylands across the world.

The major goal of this study is the cost reduction of a small-size wind turbine. In the two-year project consigned from Tottori prefecture, the target cost of energy of the $7 \mathrm{~m}$-BWT system for grid connection was about $26 \mathrm{yen} / \mathrm{kWh}$ (about $24 \mathrm{cent} / \mathrm{kWh}$ ) at the site of annual average wind speed of $5 \mathrm{~m} / \mathrm{s}$. Although the high purchase price ( $55 \mathrm{yen} / \mathrm{kWh}$ without tax) for a small wind-power facility had been included in the Japanese feed-in tariff (FIT) [23] that started in July 2012, the category of small wind power was integrated into that of large wind power in April 2018. In order to cope with the change in the situation and to promote the introduction of small wind power in low wind-energy regions (annual average wind speed: $3.5 \mathrm{~m} / \mathrm{s}$ ), another plan has just been started, in which the rotor diameter of the BWT equipped with the OCS will be enlarged to $12.5 \mathrm{~m}$ with no change in the power-generation system, according to the concept, so as to reduce the cost of the energy. The OCS will be improved to be given a simpler and tougher structure than the present one in response to the enlargement of the rotor diameter.

\section{Conclusions}

The development of the $7 \mathrm{~m}$-diameter BWT equipped with a mechanical OCS was described in this paper. The shape of the guide grooves, which were the main parts of the OCS twisting the blades by centrifugal force, was improved by the pre-compression of the springs. By considering the observed radial force from which the centrifugal force was excluded, the prediction of twist angle variation against the rotor rotational speed agreed to some extent with the results of the forcible rotation experiments that used an induction motor. From the predicted behavior of the twist angle, the rated rotational speed of the BWT was assumed to be $110 \mathrm{rpm}$. In this study, a coreless generator was combined with a speed-increasing planetary gear unit and the performance of the BWT equipped with the geared generator was predicted based on the BEM theory with the QMS model. It was predicted that the BWT rotor could be revolved at the rated rotational speed with the twist angle of $12^{\circ}$ in wind speed of about $13 \mathrm{~m} / \mathrm{s}$, i.e., a rated wind speed. Moreover, the rotor was predicted not to be able to 
rotate the generator which was controlled to allow the smallest external resistance of $24 \Omega$ in cases over the rated wind speed due to the reduction of rotor torque by the large inclination of blades. Despite the smaller generator capacity compared with the case without the OCS, the prediction of the AEP of the BWT with the OCS gave $5791 \mathrm{kWh}$, a little less than the case without the OCS, for the annual average wind speed of $5 \mathrm{~m} / \mathrm{s}$. The relations obtained in the power-generation experiments between the electric power and the wind speed and between the rotor speed and the wind speed coincided reasonably with the BEM-based predictions. The 5-min averaged power of more than $2 \mathrm{~kW}$ was measured, which suggested that the special power-generation control system using two controllers and one power-conditioning system worked well without significant problems. Under the conditions of rather strong wind in the generation experiments, the maximum rotor speed of $107.3 \mathrm{rpm}$ was measured following the observation of the maximum wind speed of $18.9 \mathrm{~m} / \mathrm{s}$ and, subsequently, the large twist motion of the blades was confirmed. In that twist motion observed during the generation experiments, the time to restore to the original state from the twisted state of the blades was much shorter than that needed in the forcible rotation experiments. In order to obtain more data regarding power generation, moving the present BWT system to another site where better wind conditions can be expected is now planned as the next stage.

Supplementary Materials: The following video is available online at http://www.mdpi.com/2411-9660/2/2/17/s1, Video S1: Manual motion test of the OCS used in the second forcible rotation experiments and observation of the first twist motion of the BWT equipped with the OCS in strong wind conditions on 1 March 2018.

Author Contributions: Y.H. carried out the fundamental design of the BWT and OCS, predicted the performance, and wrote this paper. K.T. (Kotaro Tagawa) contributed to fund acquisition, considered the application of the butterfly wind turbine to drylands, and proofread this paper. S.S. (Shigenori Saito) developed the concept and the turbine blades. K.S. developed the turbine blades and the over-speed control system. T.O. developed the tripod-type stand and proposed the idea of the pre-compression of springs. K.M. and K.T. (Kazutoshi Toba) prepared the experiments and conducted the data acquisition. T.H. developed the geared coreless generator. Y.T. developed the generation controller and the software. K.T. (Kazuo Takashima) and S.S (Susumu Sasaki). developed the control console. K.N. investigated the spring constant and conducted the strength test of the universal joints. S.Y. gave comments on the numerical analysis of the turbine blades, discussed the VAWT with other authors, and revised the paper.

Funding: This research was funded by the Tottori Prefecture Project for Promoting Collaborative Commercialization between Industry and Academia and was supported in part by the International Platform for Dryland Research and Education (IPDRE), Tottori University, and the Collaborative Research Program of the Research Institute for Applied Mechanics, Kyushu University.

Acknowledgments: The authors are profoundly grateful for the cooperation of Diamond Electric Mfg. Co., Ltd., Matsumura Seiki Inc., and Nikkei Technology Center Co., Ltd. This project was supported by many staff at the Organization for Regional Industrial Academic Cooperation, Tottori University.

Conflicts of Interest: All authors declare no conflict of interest.

\section{Appendix A}

Figures A1 and A2 show the non-dimensional torque and power of the BWT predicted by the BEM theory, respectively. The torque coefficient $C_{q}$ is defined by $Q /\left(0.5 \rho A_{0} V_{\infty}{ }^{2} R\right)$. The power coefficient $C_{p}$ is defined by $Q \omega /\left(0.5 \rho A_{0} V_{\infty}{ }^{3}\right)$ and the tip speed ratio $\lambda$ is defined by $R \omega / V_{\infty}$. Here, $\rho$ is the air density. $A_{0}$ is the swept area in the no-twist state and is assumed to be constant regardless of the twist angle $\eta$. Of course, the change in the swept area $A$ of the BWT rotor depending on the twist angle is considered in the calculation of torque $Q$. In the same manner as the calculations for Figures 11 and 12, the QMS [12] was used as the streamtube model and the principal aerodynamic data were introduced from the reference $[15,16]$. From the features of the BWT, the blade tip loss and the arm effects, which are significant in general straight-bladed VAWTs, are not considered in Figures A1 and A2. In addition, the effects of turbulence, rotor hub, and stand are not included. Needless to say, the influence of the slant blade portions, i.e., the inner rotor, is considered by the QMS model. The maximum power coefficient of 0.415 is predicted at the tip speed ratio of 3.7 in the case of $8 \mathrm{~m} / \mathrm{s}$ without twist. 


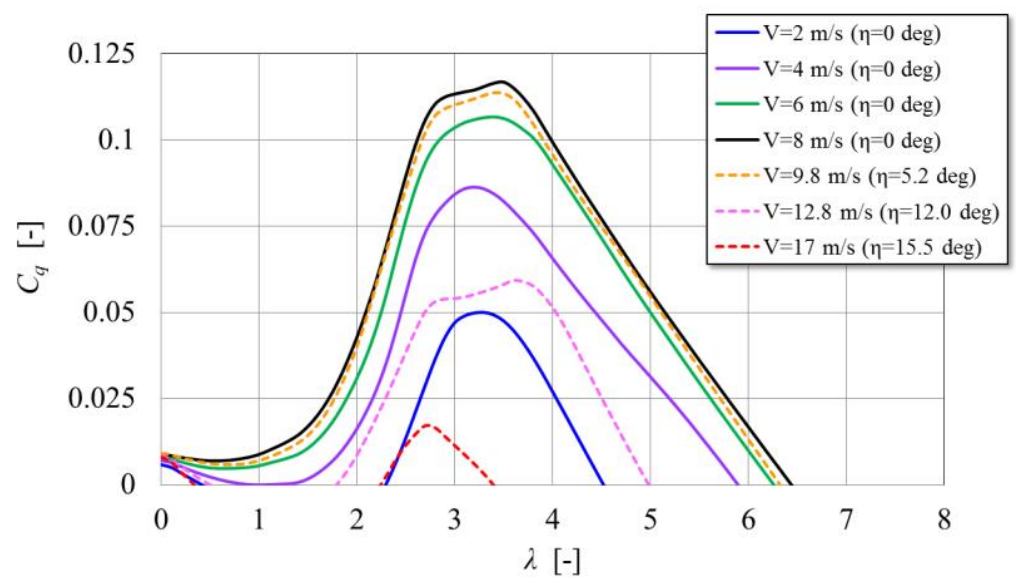

Figure A1. Non-dimensional torque of the BWT predicted by the BEM theory.

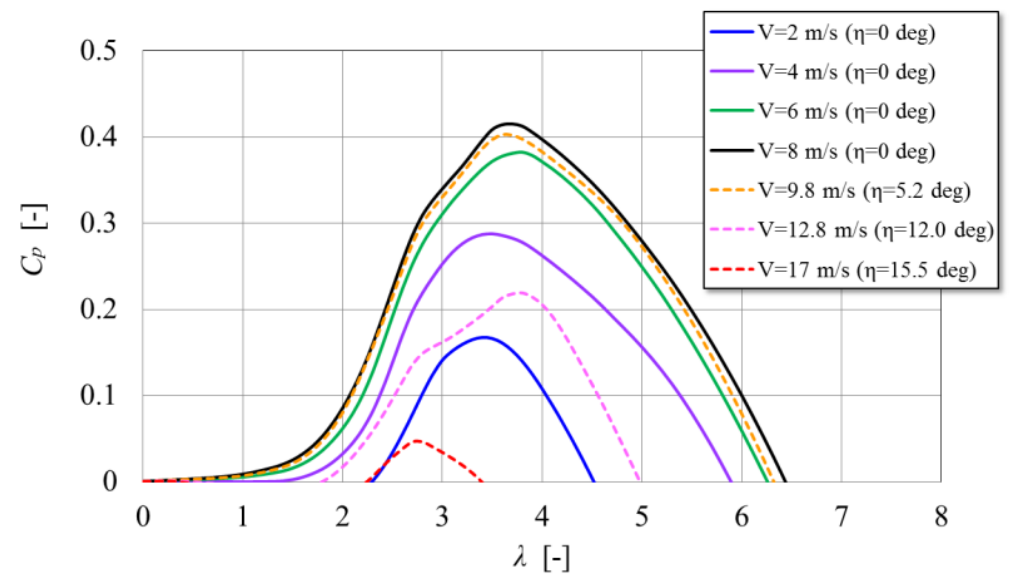

Figure A2. Non-dimensional power of the BWT predicted by the BEM theory.

\section{References}

1. GWEC, Global Statistics. Available online: http://gwec.net/global-figures/graphs/ (accessed on 9 May 2018).

2. U.S. Department of Energy's Office of Energy Efficiency and Renewable Energy (EERE). Environmental Impacts and Siting of Wind Projects. Available online: https:/ /www.energy.gov/eere/wind/environmentalimpacts-and-siting-wind-projects (accessed on 9 May 2018).

3. Van Bussel, G.; Mertens, S. Small wind turbines for the built environment. In Proceedings of the Fourth European \& African Conference on Wind Engineering (EACWE4), Prague, Czech Republic, 11-15 July 2005; Naprstek, J., Fischer, C., Eds.; Paper \#210. ITAM AS CR: Prague, Czech Republic, 2005.

4. Mertens, S.; van Kuik, G.; van Bussel, G. Performance of an H-Darrieus in the skewed flow on a roof. J. Sol. Energy Eng. 2003, 125, 433-440. [CrossRef]

5. Goude, A.; Rossander, M. Force Measurements on a VAWT Blade in Parked Conditions. Energies 2017, 10, 1954. [CrossRef]

6. Hara, Y.; Sumi, T.; Emi, T.; Yokoyama, M.; Akimoto, H.; Kawamura, T.; Nakamura, T. Effects of blade section on performance of butterfly wind turbines as double-blade VAWTs. JFST 2015, 10. [CrossRef]

7. Hara, Y.; Shiozaki, A.; Nishiono, H.; Saito, S.; Shioya, K.; Sumi, T.; Matsubara, Y.; Yasumoto, Y.; Takagaki, K.; Kogo, S. Experiment and numerical simulation of an aluminum circular-blade butterfly wind turbine. JFST 2016, 11. [CrossRef]

8. Hara, Y.; Tagawa, K. Butterfly Wind Turbines with Mechanical Over-speed Control System. In Proceedings of the 1st International Symposium on Wind and Tidal Power (ISWTP 2017), Montreal, QC, Canada, 28-30 May 2017; Paraschivoiu, I., Dy, N.V., Eds.; IOPARA Inc.: Montreal, QC, Canada, 2017; pp. 1-8. 
9. Gipe, P. Wind Power for Home E Business; Chelsea Green Publishing: Totnes, UK, 1993; pp. 133-146, ISBN 0-930031-64-4.

10. Yamada, T.; Kiwata, T.; Kita, T.; Hirai, M.; Komatsu, N.; Kono, T. Overspeed Control of a Variable-Pitch Vertical-Axis Wind Turbine by Means of Tail Vanes. J. Environ. Eng. 2012, 7, 39-52. [CrossRef]

11. Hansen, M.O.L. Aerodynamics of Wind Turbines; James \& James (Science Publishers) Ltd.: London, UK, 2000; pp. 48-59. ISBN 1-02916-06-9.

12. Paraschivoiu, I. Wind Turbine Design: With Emphasis on Darrieus Concept; Polytechnic International Press: Montreal, QC, Canada, 2002; pp. 147-259. ISBN 2-553-00931-3.

13. Hara, Y.; Kawamura, T.; Akimoto, H.; Tanaka, K.; Nakamura, T.; Mizumukai, K. Predicting Double-Blade Vertical Axis Wind Turbine Performance by a Quadruple-Multiple Streamtube Model. IJFMS 2014, 7, 16-27. [CrossRef]

14. Hara, Y.; Saito, S.; Shioya, K.; Shiozaki, A.; Nishiono, H. A proposal on a Compact Mechanics of Over-Speed Control for Vertical Axis Wind Turbines. In Proceedings of the 36th Symposium of Wind Energy Utilization, Tokyo, Japan, 27-28 November 2014; pp. 389-392.

15. Sheldahl, R.E.; Paul, C.K. Aerodynamic Characteristics of Seven Symmetrical Airfoil Sections through 180-Degree Angle of Attack for Use in Aerodynamic Analysis of Vertical Axis Wind Turbines; SAND-80-2114; Sandia National Laboratories: Albuquerque, NM, USA, 1981; p. 120.

16. Kumar, V.; Paraschivoiu, M.; Paraschivoiu, I. Low Reynolds Number Vertical Axis Wind Turbine for Mars. Wind Eng. 2010, 34, 461-476. [CrossRef]

17. Naoko, I. A Study on CFD Analysis of Aerodynamic Data of Airfoils for Prediction of Performance of VAWTs. Master's Thesis, Tottori University, Tottori, Japan, 2013.

18. Murata, J.; Maeda, T.; Kamada, Y.; Ogasawara, T.; Shimizu, K. Development and Validation of Analysis Method for Straight-Bladed VAWT. In Proceedings of the 37th Symposium of Wind Energy Utilization, Tokyo, Japan, 26-27 November 2015; pp. 361-364.

19. Hara, Y.; Saito, S.; Shioya, K.; Shiozaki, A.; Nishiono, H.; Okutani, M.; Mishima, K.; Kawabata, T.; Yoshida, S. Over-speed Control System for Vertical Axis Butterfly Wind Turbine. J. Wind Energy JWEA 2017, 41, 9-16.

20. Burton, T.; Sharpe, D.; Jenkins, N.; Bossanyi, E. Wind Energy Handbook, 1st ed.; John Wiley \& Sons, Ltd.: West Sussex, UK, 2001; pp. 14-16. ISBN 0-471-48997-2.

21. Hokuei Takachiho Solar Power Plant. Available online: http://enatex.sanin.jp/p/enatex/2/3/7/ (accessed on 13 May 2018).

22. Arid Land Research Center, Tottori University. Available online: http://www.alrc.tottori-u.ac.jp/english/e_ index.html (accessed on 14 May 2018).

23. Feed-In Tariff Scheme in Japan. Available online: http://www.meti.go.jp/english/policy/energy environment/renewable/pdf/summary201207.pdf (accessed on 14 May 2018). 